\title{
Relaxation of silicon non-equilibrium depletion with majority charge carriers in strong electric fields, its mechanisms and ways to damp it
}

\author{
Dedicated to the $80^{\text {th }}$ anniversary of Academician O.V. Snitko \\ V.E. Primachenko, S.I. Kirillova and V.A. Chernobay \\ V. Lashkaryov Institute of Semiconductor Physics, NAS of Ukraine, \\ 41, prospect Nauky, 03028 Kyiv, Ukraine \\ E-mail: pve18@isp.kiev.ua
}

\begin{abstract}
This overview deals with original works of authors as well as with works by native and foreign authors, which are devoted to this or close topics. It is written with account of the modern state of the problem, to solve which a great amount of successful work was made by Academician of NAS of Ukraine O.V. Snitko. Considered in this paper are the essence of the phenomenon of non-equilibrium depletion with majority charge carriers that takes place in the field effect in silicon and a set of new phenomena that were revealed by the authors when investigating this effect. Besides, analyzed in detail are the processes of acceleration inherent to relaxation of the non-equilibrium depletion in strong electric fields $\left(E_{s}=3 \cdot 10^{3}-5 \cdot 10^{5} \mathrm{~V} / \mathrm{cm}\right)$ at silicon surface being in various physical-and-chemical states. It is noted that the Franz-Keldysh or Frenkel effects for local centers play the main role in acceleration of relaxation with growing $E_{s}$ at the silicon surface. In this case, an essential role belongs to electron-phonon interaction of the charge carrier at the local center with continuum phonons around this center.
\end{abstract}

Keywords: field effect, non-equilibrium depletion, strong electric field.

Manuscript received 17.06.08; accepted for publication 20.06.08; published online 30.09.08.

\section{Introduction}

The phenomenon of semiconductor conductance change under influence of external electric field applied to semiconductor surface through a dielectric spacing got the brief title "field effect" [1-8]. This change of semiconductor conductance takes place as a result of introduction (or taking out) of charge carriers (electrons or holes) into it by using an electric circuit that consist of a voltage source, leads and capacitor created from semiconductor, dielectric and conducting (most often metal) electrode.

As far as in 1948 W. Shockly and G.L. Pearson [9] looked to change the conductance of thin silicon and cooper oxide films by using external electric field to increase the current flowing through them. However, they did not obtain any essential result, which was explained by authors as screening the external field by a charge that after introduction into semiconductor was caught at surface electron states (SES) in semiconductor and could not take part in conductance as it takes place when it is in the subsurface region - region of the space charge (SCR).
Devices based on the field effect - the so-called "field effect transistors" - got wide application both as single elements and in the composition of integrated circuits only after applying the silicon as semiconductor, on which a dielectric spacing $\left(\mathrm{SiO}_{2}\right)$ was created by thermal oxidation of silicon surface $[10,11]$. Since that time, the investigations of $\mathrm{Si}-\mathrm{SiO}_{2}-\mathrm{Me}$ (metal) systems were widely performed with various silicon parameters, $\mathrm{SiO}_{2}$ states and its boundary with silicon where possible are various defects of structural and energetic character, including boundary electron states (BES). The so-called "real" surface without a thermally grown $\mathrm{SiO}_{2}$ film was under thorough investigation, too, with the aim to preliminary prepare silicon surface to thermal oxidation as well as with the purpose to reveal various phenomena at the surface and in the subsurface region to determine parameters of the latter in different physical-andchemical states of the surface (atomically clean, naturally oxidized in ambient air, treated with various etching agents, doped with impurities of other elements, in particular, metals, etc.). As a consequence, a plethora of works was published, results of which were partially analyzed in monographs [1-8] and [12-16]. 


\section{Non-equilibrium depletion of silicon with majority charge carriers in the field effect}

It is known that in the conditions of thermodynamic equilibrium the external electric field changes the silicon conductance rather weakly at the cost of changes in electron and hole concentrations near surface and penetrates not so deeply into semiconductor (only of the order of one micrometer or less) [17]. In this case, except the change of carrier concentration, possible is the change of their mobility caused by scattering at silicon surface, which for the first time was considered by J.R. Schriffer [18]. At the same time, the main part of the charge induced by the field in semiconductor is chiefly in SES (especially at the thermally non-oxidized silicon surface), which causes screening the semiconductor bulk from field influence.

In the non-stationary regime of the field effect that, as a rule, is realized immediately after turning the field on, when the thermodynamic equilibrium is absent both between c- and v-semiconductor energy bands as well as between these bands and local levels in SCR and at the surface (SES). In practice, only the concentration of majority charge carrier changes in semiconductor during the time of charging the capacitor made with this semiconductor. When the capacitor voltage polarity corresponds to enriching semiconductor with the majority carriers, charging the capacitor as well as reaching the thermodynamic equilibrium between energy bands and local levels (that partially capture the introducted charge) happen relatively fast (except trapping the charge into the socalled "slow" SES) as it is not necessary to spent the activation energy on charge carrier transitions [2-5]. Another situation takes place when applying to the capacitor the voltage with the sign providing the depletion of semiconductor with the main charge carriers as a result of their taking out from semiconductor. Realized in this case is the thermodynamically non-equilibrium field effect when for a long time the thermodynamic equilibrium between cand v-bands and local levels (including SES and BES) is absent. It is associated with energy expenses on activation (generation) of carriers during transitions between bands and levels.

In our works [19-21], for the first time we investigated far and wide the phenomenon of nonequilibrium depletion with the majority charge carriers, which takes place in silicon wafers of $p$ - and $n$-types of conductance with a real (treated with an etching agent) surface. In these experiments, we used both depleting fields and turning off the enriching ones. In the latter case, silicon depletion was caused by the charge trapped in SES after applying the enriching fields. The values of the applied fields were varied within a wide range, which enabled us to reveal for the first time the following effects: 1) full taking out of charge carriers form the plates with the thickness close to $300 \mu \mathrm{m}$ (current through them was decreased practically to zero;
2) growth and, after reaching some definite field values, decrease in the relaxation time of non-equilibrium depletion as well as time of samples being in a nonconducting state (time of current cut-off), which means field acceleration of the relaxation time for these effects; 3 ) dependences of the relaxation time as well as current cut-off time on temperature within the range 300 to $170 \mathrm{~K}$; 4) essential decrease in relaxation and current cut-off times (or even vanishing the latter) in dependence on exposure with white light.

It is noteworthy that relaxation of non-equilibrium depletion in relatively low fields (that do not accelerate it) was studied earlier in silicon with the aim to determine parameters of SES that exchange the majority carriers with its band [22, 23]. Realized in [24] was the phenomenon of non-equilibrium depletion with the majority carriers in germanium wafers that were placed into cryostat at low temperatures (close to $77 \mathrm{~K})$.

In [25] at $77 \mathrm{~K}$, using the non-equilibrium depletion, determined were the following silicon parameters: surface potential (band bend) without any applied voltage $(V=0)$, concentration values for charge carriers and their mobilities, while in [26] the method of nonequilibrium depletion was used to determine parameters of the intervalley scattering for electrons in $n$-Si within a subsurface region.

Investigated in [19-21] were silicon samples both of $n$ - and $p$-types. Data obtained using $n$-Si $(\rho=$ $200 \mathrm{Ohm} \cdot \mathrm{cm}$ ) had the same qualitative character as compared with those obtained for the samples $p$-Si $(\rho=$ $4100 \mathrm{Ohm}^{\circ} \mathrm{cm}$ ) that were subjected to the most full study. Sizes of the samples were as follows: $1.2 \times 0.5 \times(0.02-0.05) \mathrm{cm}$. Most of the samples had special block creations on their sides to avoid the external electric field at the deposited metal (Au) contacts. The measurements were performed in a vacuum cryostat $\left(\sim 1 \cdot 10^{-4} \mathrm{~Pa}\right)$ within the temperature range $T=300-170 \mathrm{~K}$ with the external electric field in the form of П-pulses of varied duration and amplitude, both single and train-like with a variable frequency.

Summarized in Fig. 1 are the dependences of nonequilibrium conductance on the voltage applied to the metal electrode $V_{\mathrm{M}}$ for several measurement temperatures of $p$-Si. The conductance was measured since $\sim 100 \mu$ s after turning $V_{\mathrm{M}}$ on, which by its time was enough to charge the field capacitor. It is seen from Fig. 1 that positive $V_{\mathrm{M}}$ provides great changes (decrease) in conductance. As was mentioned above, it is caused by the absence of SES screening action as a consequence of deferred exchange of carriers with extended silicon bands. As a result, the field penetrates into silicon rather deep and at $V_{\mathrm{M}}=400 \mathrm{~V}$ extracts holes from it all over (conductance "cut-off"). When $T=298 \mathrm{~K}$, and the exchange rate for carriers between SES and bands increases, the dependence $\sigma\left(V_{\mathrm{M}}\right)$ has the "ordinary" equilibrium character with the minimum, which is indicative of a created inversion electric layer at $V_{\mathrm{M}} \geq 400 \mathrm{~V}$. 

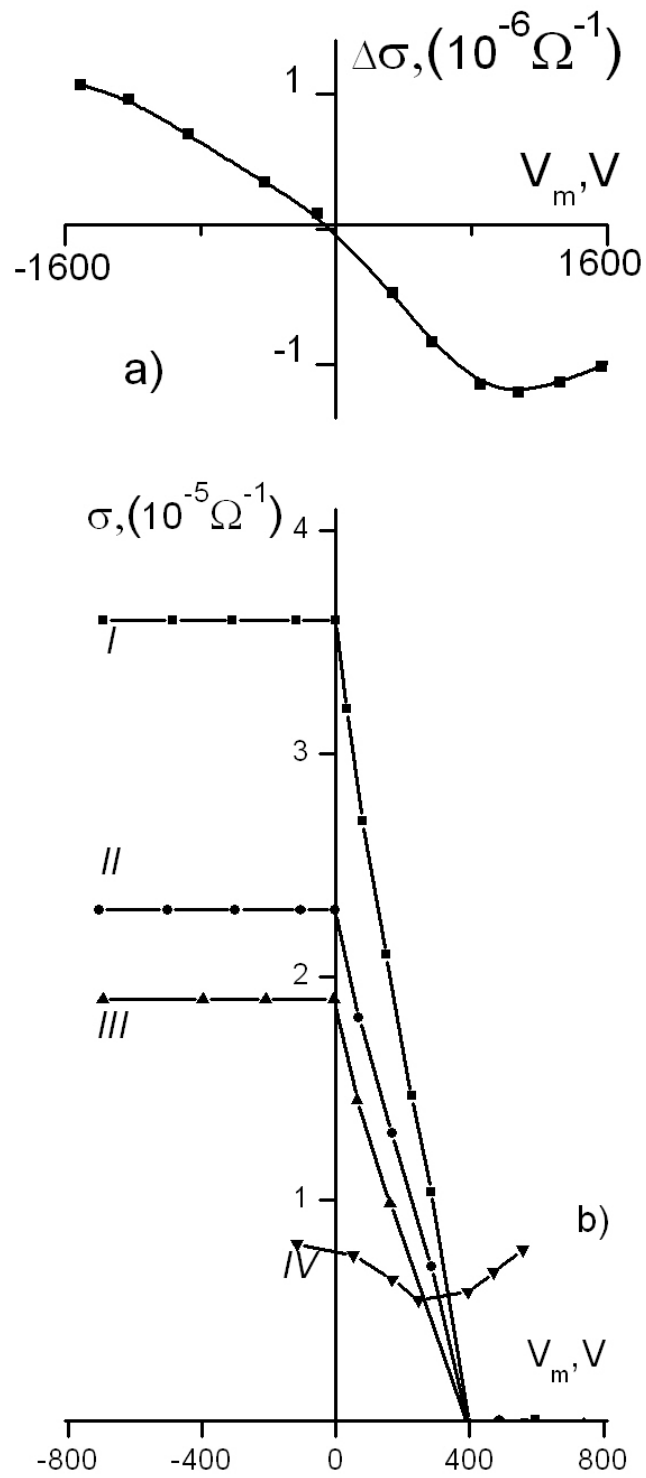

Fig. 1. a) Equilibrium conductance vs the constant external electric voltage $V_{\mathrm{M}}(T=184 \mathrm{~K}, p-\mathrm{Si}, \rho=4100 \mathrm{Ohm} \bullet \mathrm{cm})$. b) Non-equilibrium conductance vs $V_{\mathrm{M}}$ for four temperatures (I - 184, II - 223, III - 241, IV - $298 \mathrm{~K})$. The conductance measured in $100 \mu$ s after applying $V_{\mathrm{M}}$ was calculated per $1 \mathrm{~cm}^{2}$ of the surface.

Fig. 2 shows typical oscillographic pictures for the non-equilibrium field effect at low temperatures. In this case, relaxation has a complex non-exponential character, and therefore it is characterized with effect halfdecay time $\tau_{1 / 2}$. This non-exponential decay indicates that realization of thermodynamic equilibrium in $p$-Si is not limited by activation transitions between $\mathrm{v}$-band and a single local SES, as it was adopted for low $V_{M}[22,23]$. At $V_{\mathrm{M}}>+400 \mathrm{~V}$, there observed the current "cut-off" in the sample. The cut-off time is characterized with the value $\Theta$. Shown in Fig. 3 are the dependences $\tau_{1 / 2}\left(T^{-1}\right)$ and $\Theta\left(T^{-1}\right)$. They have two practically exponential parts (at $T>210 \mathrm{~K}$ and $T<210 \mathrm{~K}$ ), which is indicative of the fact that at least two discrete SES take part in relaxation.
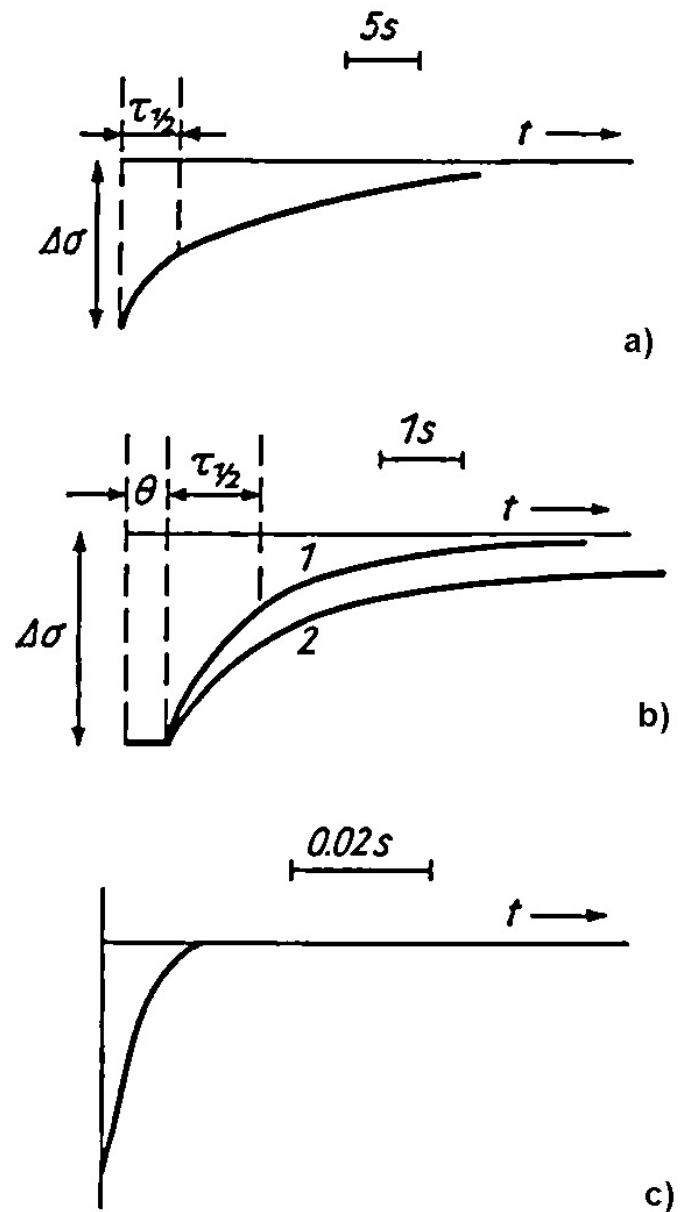

c)

Fig. 2. Typical oscillograms of the non-equilibrium depletion effect at the temperature $171 \mathrm{~K}$ when applying $V_{\mathrm{M}}, \mathrm{V}$ : +200 (a); +900 (b, 1); -900 (b, 2); +900 (c, light injection $\left.\Delta p / p_{0}=0.005\right), T=171 \mathrm{~K}, p-\mathrm{Si}$.

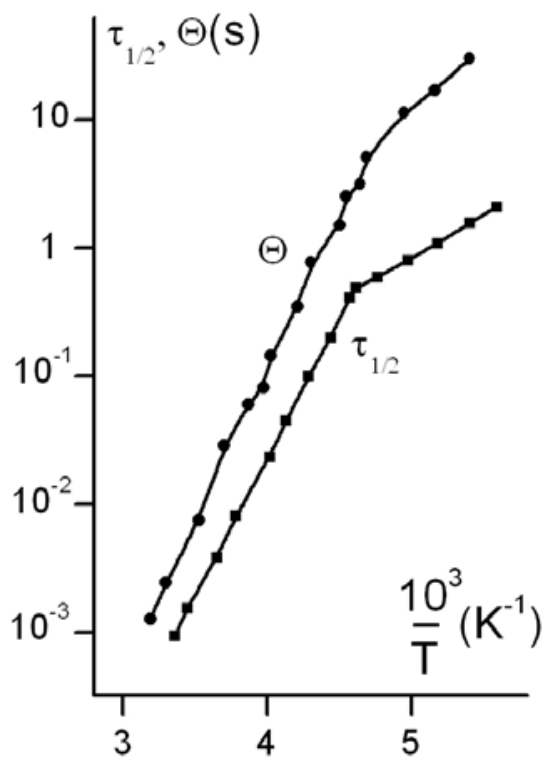

Fig. 3. Temperature dependences for times of the conductance half-decay $\tau_{1 / 2}$ and cut-off $\Theta\left(p-\mathrm{Si}, \Delta V_{\mathrm{M}}=+400 \mathrm{~V}\right)$. 
When applying to $p$-Si the negative voltage $\left(-V_{\mathrm{M}}\right)$ that enriches $p$-Si with holes, the change of conductance $\sigma$ is several hundred times less than at $+V_{\mathrm{M}}$, with the conductance fast relaxing (in microseconds) down to the quasi-stationary values (Fig. 1) that are practically unchanged within the figure scale. It is caused by capture of holes in SES, which results in screening the field, and the latter does not penetrate into $p$-Si bulk. After turning $-V_{\mathrm{M}}$ off, the hole charge captured in SES at $-V_{\mathrm{M}}$ causes qualitatively the same non-equilibrium depletion of $p$-Si by holes as it takes place when turning $+V_{\mathrm{M}}$ on. And, respectively, turning $+V_{\mathrm{M}}$ off causes the effect identical to turning $-V_{\mathrm{M}}$ on.

Presented in Fig. 4 dependences $\tau_{1 / 2}\left(V_{\mathrm{M}}\right)$ and $\Theta\left(V_{\mathrm{M}}\right)$ corresponds to applying the voltages $V= \pm V_{\mathrm{M}}$ at $T=171 \mathrm{~K}$. These dependences were obtained for a larger thickness of dielectric in the capacitor from $p$-Si (with mica), therefore, the cut-off was observed not at $+400 \mathrm{~V}$ but at $+480 \mathrm{~V}$. At the initial stages, when $+V_{\mathrm{M}}$ increases up to $+100 \mathrm{~V}$ one can observe the growing $\tau_{1 / 2}$ with $+V_{\mathrm{M}}$ (curve 1). It is associated with non-equilibrium conditions in $p$-Si bulk and agrees with data of [23]. In this case, $\tau_{1 / 2}$ value is determined by the rate of electron transitions from v-band to SES as a consequence of their thermal excitation, which results in growth of $p$-Si conductance due to increasing the hole concentration in the space charge region. When $V_{\mathrm{M}}>+100 \mathrm{~V}$, beside thermal excitation, the electric field at $p$-Si surface creates supplementary conditions to accelerate relaxation of the effect of SCR depletion by holes, and the time $\tau_{1 / 2}$ decreases.

The same character is inherent to the dependence $\tau_{1 / 2}\left(V_{\mathrm{M}}\right)$ for relaxation of a post-action $\left(-V_{\mathrm{M}}\right)$ when the magnitudes $\left|V_{\mathrm{M}}\right|$ correspond to those before cut-off (curve 3 ). The values of $\tau_{1 / 2}$ different to some extent as compared with the curve 1 can be explained by the following: 1) in the process of post-action relaxation for negative $V_{\mathrm{M}}$, the efficient capacitance increases, that is the charge induced in semiconductor grows; in the

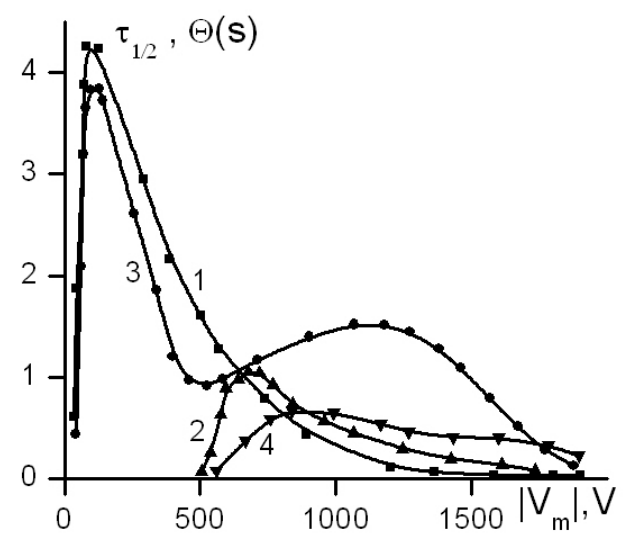

Fig. 4. Dependence of times $\tau_{1 / 2}$ and $\Theta$ on the value $V_{\mathrm{M}}$. $1-\tau_{1 / 2} ; 2-\Theta\left(\right.$ at $\left.+V_{\mathrm{M}}\right) ; 3-\tau_{1 / 2} ; 4-\Theta$ for post-action $\left(-V_{\mathrm{M}}\right) \cdot p-\mathrm{Si}, T=240 \mathrm{~K}$. course of post-action $\left(-V_{\mathrm{M}}\right)$ it decreases with time; 2) charge in surface states after turning the negative voltage off (when the metal electrode is connected with ground) is screened not only by the charge of the opposite sign in the semiconductor bulk, but the charge of the opposite sign on the metal electrode, which lowers the induced charge in the semiconductor bulk; 3) capture of the charge by surface states, and in this case conductance relaxation is realized by different ways for $+V_{\mathrm{M}}$ action and $-V_{\mathrm{M}}$ post-action due to different filling the states. The opportunity of realization of these reasons is confirmed, for instance, by some lower values of negative voltage post-action as compared with the field effect for the positive voltage of respective magnitudes.

The time, during which the semiconductor has practically no conductance at applied $+V_{\mathrm{M}}$ ("cut-off time" $\Theta$ ), possesses its peak value at definite voltage magnitudes (curve 2). The rate of $\Theta$ growth up to its peak value with increasing the voltage is higher as compared with the rate of its drop at high voltages. This dependence can be explained by both thermal and field generation of carriers. At higher temperatures, strong fields (for example, $V_{\mathrm{M}} \geq 1200 \mathrm{~V}$ at $241 \mathrm{~K}$ ) can decrease $\Theta$ down to the time comparable with that of charging the capacitor. Therefore, the cut-off is not practically observed.

In the case of $-V_{\mathrm{M}}$ post-action (curve 4 ), the dependence of $\Theta$ on the voltage has a peak, too, but the latter is shifted into the region of higher $\left|V_{\mathrm{M}}\right|$, and the value $\Theta_{\max }$ is lower a little than in the dependence 2. When $\left|V_{\mathrm{M}}\right|>900 \mathrm{~V}, \Theta$ of post-action has higher values than those for the respective $+V_{\mathrm{M}}$, and its dependence within the range of voltages $1000-1800 \mathrm{~V}$ is more smooth. Probably, it is caused by the same reasons that yield in differences between $\tau_{1 / 2}$ values for $+V_{\mathrm{M}}$ action and $-V_{\mathrm{M}}$ post-action. And finally, growth of post-action $\tau_{1 / 2}$ for negative $V_{\mathrm{M}}$ within the range $500-1500 \mathrm{~V}$ in comparison with $\tau_{1 / 2}$ that corresponds to the voltage close to $500 \mathrm{~V}$ is caused by peculiarities of post-action relaxation. As was said above, the matter is that post-action relaxation is non-exponential: for larger time intervals after turning the voltage off the instantaneous time of effect relaxation is increased. Within the area of cut-off appearance $\left(V_{\mathrm{M}}>|500| \mathrm{V}\right) \tau_{1 / 2}$ was measured after the time $\Theta$, and not immediately after turning the field off as it was done in the cases of voltages less than $500 \mathrm{~V}$. It is the reason that causes higher $\tau_{1 / 2}$ values. The reasons of discrepancies between $\tau_{1 / 2}$ values within the ranges $V_{\mathrm{M}}<|500| \mathrm{V}$ and $V_{\mathrm{M}}>|500| \mathrm{V}$ for the cases of $+V_{\mathrm{M}}$ action and $-V_{\mathrm{M}}$ post-action are the same. For example, Fig. $2 \mathrm{~b}$ shows the oscillograms of $+V_{\mathrm{M}}$ action and $-V_{\mathrm{M}}$ post-action in the area of cut-off; in the case of $-V_{\mathrm{M}}$ post-action, conductance relaxation is decelerated especially after the half-decay time.

The non-equilibrium field effect takes place at the room temperature, too. It is seen from Fig. 1 (curve 4) that, although the cut-off is not observed in this case, the conductance minimum is rather deep (one third of the total sample conductance). While the depth of the

(C) 2008, V. Lashkaryov Institute of Semiconductor Physics, National Academy of Sciences of Ukraine 
equilibrium minimum (measurements in 2 to $3 \mathrm{~ms}$ after turning the field on) at the room temperature is tens of times less, with the minimum corresponding to the same voltage as that for the non-equilibrium one.

It should be noted that light has an essential influence on the non-equilibrium field effect. It should be expected, as light generates electron-hole pairs and causes transitions between levels and bands, which accelerates the course of the non-equilibrium process. Shown in Fig. 2 are the set of oscillograms that demonstrate the influence of light exposure providing the injection level of the order of $\Delta p / p=0.005$. They are obtained at $+800 \mathrm{~V}$ and the temperature $170 \mathrm{~K}$. The oscillogram (a) was obtained with the darkened sample, while (c) - under constant light exposure. It is seen that the time of the effect half-decay is almost 200 times decreased under exposure. Besides, the cut-off is not practically observed in this case, although in darkness the cut-off duration is close to $1 \mathrm{~s}$.

The oscillogram (b) describes field effect relaxation for the case of pulse periodical light exposure with the same injection level. Oscillogram hatches show conductance relaxation in darkness, and transitions between hatches corresponds to periods of sample exposure. Note that at the initial moment (after turning the field on and charging the capacitor) the effect magnitude is the same in all the cases. It means that the field penetrates over the total semiconductor thickness. Light considerably accelerates the effect relaxation. The latter depends not only on the light intensity (injection level) but on the duration of its action. At higher light intensities, relaxation processes naturally accelerate. Thus, investigation of the non-equilibrium field effect needs thorough darkening the sample, which was provided.

When investigating the non-equilibrium depletion with majority carriers, it is necessary for contacts to be non-injecting of minority carriers, as in the opposite case this also results in acceleration or liquidation of the effect.

Thus in the work [19], investigated for the first time was the non-equilibrium depletion in strong electric fields. This enabled to reveal such phenomena as: cut-off of the current through the sample, relaxation acceleration of the non-equilibrium depletion due to field mechanisms, essential influence of the temperature and white light exposure on the processes under investigation.

In $[20,21]$, being based on the data above the authors performed theoretical verification of the field effect mechanism in the regime of non-equilibrium depletion. They calculated changes of the space charge in SCR, electric field in $p$-Si, $\mathrm{Si}$ conductance and capacitance in the dependence of the potential drop on it in the non-equilibrium regime. It is shown that the calculated depth of field penetration at the moment of cut-off for the current through the semiconductor coincides with the $\mathrm{Si}$ sample thickness, while the calculated value of the charge carrier mobility in the field effect coincides with the hole drift mobility under high voltages, if the change in capacitance is taken into account. It is indicative of a good qualitative and quantitative accordance between the theoretical model and experimental data and confirms the offered mechanisms taking into account the dominant role of majority charge carriers in changing the conductance as well as slowing there generation time down at lowered temperatures from SES. While generation of majority charge carriers from local levels in the silicon bulk and that of minor carriers (with creation of the inversion layer) play essentially more weak role.

Being based on investigations of the nonequilibrium field effect in silicon, in [19-21] we brought out the idea to design new type of semiconductor devices with an isolated field electrode [27]. It was noted that these devices could provide amplification, modulation and storage of electric signals, realize modulation of infra-red light, play the role of a changeable resistance and capacitor (varactor), be sensitive detector of light and other radiation types, be used as time and temperature relays.

The so-called "charge coupling devices" (CCD) turned out to be the most efficient ones among those using the phenomenon of non-equilibrium depletion. For the first time, they were developed in 1970 [28, 29]. They are based on the principle of "charge coupling" in the integrated MDS system, where charge information is kept in elementary MDS cells in the regime of nonequilibrium depletion, is transferred between these cells over this integrated system by using the soft created external electric field and then withdrawn from this integrated circuit. The most successful application of CCDs is realized in optoelectronic image detectors [14]. Also developed were various types of CCDs $[8,12,14]$ that are widely used in technique.

An important property of these devices is storage of information in MDS cells and its safety in transfer, which can be disturbed by generation-recombination processes most often caused by non-equilibrium depletion. Therefore, studied in many works are the relaxation processes associated with the non-equilibrium depletion effect in silicon, which is important both for integrated MDS electronics in the whole and for ascertaining the relaxation mechanisms as well as opportunities to control them. The latter purpose can be reached in the best way, if one uses silicon and the system $\mathrm{Si}-\mathrm{SiO}_{2}$ with various physical-and-chemical surface states both in silicon and in its interface with $\mathrm{SiO}_{2}$.

\section{Mechanisms of non-equilibrium field effect relaxation in silicon under strong electric fields}

In this chapter, we are mainly focused on the results of studying non-equilibrium depletion relaxation under strong electric fields when different electric-field mechanisms of its acceleration are valid. The main attention will be paid to our works performed during 1981-1986 [30-38]. 
As it can be seen from Chapter 2 as well as from many other works, in particular [39-48], generation processes under non-equilibrium depletion in the field effect can be separated into four groups: 1) thermal monopolar generation of majority charge carriers from SES (BES) into the respective band of majority carriers; 2) thermal generation from local levels into SCR; 3) bipolar generation of electrons and holes, including participation of SES and local levels in SCR, when some inverse layer of carriers form at the semiconductor surface; 4) field generation mechanisms or the combined thermo-field ones in strong electric fields present both in semiconductor and in adjacent dielectric.

Dominance of one of these mechanisms at specific temperatures and electric field values in the vicinity of semiconductor-dielectric interface depends on: 1) semiconductor nature, its forbidden band width; 2) dielectric nature; 3) physical-and-chemical state of the surface (interface) of semiconductor; 4) concentration and capture cross-section of carriers for SES (BES) and local levels in semiconductor SCR as well as in boundary dielectric. In wide-gap semiconductors (GaAs, CdS), where the concentration of local levels in SCR is high, as a rule, and the concentration of SES is mainly high, too, relaxation of non-equilibrium depletion is realized due to electron (hole) transitions between them and the respective band of majority carriers. In this case, the inversion layer is not created, as a rule, even after a long time [46-48].

Another situation takes place in narrow-gap semiconductors, in particular in silicon and its boundary with $\mathrm{SiO}_{2}$ [39-46] where possible are all (1) to (4) mentioned above processes in non-equilibrium depletion. Dominance of one of them depends, first of all, on the physical-and-chemical state of silicon surface and its SCR as well as on values of temperature and electric field. After a long time of non-equilibrium depletion relaxation, as a rule, there arises the inversion layer of minor charge carriers at the external boundary of SCR.

Mechanisms of non-equilibrium depletion relaxation in strong electric fields when thermal-and-field or pure field acceleration of relaxation were studied in our works [30-37] for various states of silicon surface, namely: atomically clean surface; real (etched) $\mathrm{Si}$ surface; Si surface after thermal oxidizing it; real Si surface after doping it with metal impurities; industrial MDS structures, including $\gamma$-irradiated as well.

It is noteworthy that in our works the calculated electric field on silicon surface being in various states, when field acceleration of non-equilibrium depletion relaxation was observed, was within the range $3 \cdot 10^{3}$ to $1 \cdot 10^{5} \mathrm{~V} / \mathrm{cm}$. These field values did not result in carrier avalanche inside SCR in our samples $[8,49,50]$. In most cases, this relaxation acceleration can be successfully explained using the works by Timashev [51,52], who considered ionization of deep centers inside SCR in semiconductors with account of the Frenkel [53] and Franz-Keldysh $[54,55]$ effects. The essence of the former one is electric-field lowering the potential barrier for releasing the charge carriers from the deep center that possesses the far-acting potential. While the FranzKeldysh effect is associated with transitions of charge carriers to virtual states in the forbidden gap, which arise near allowed bands in strong electric fields.

Investigations of non-equilibrium depletion relaxation were performed using the samples of $p$-Si ( $\rho=$ $2300 \mathrm{Ohm} \cdot \mathrm{cm})$ and $n$-Si $(\rho=170 \mathrm{Ohm} \cdot \mathrm{cm})$. After applying the voltage $V_{\mathrm{M}}$ to the measuring capacitor $\mathrm{M}$ mica-Si depleted with majority carriers and charging the capacitor by using an oscillograph with memory, we measured changes in semiconductor conductance $d \sigma_{s} / d t(t)$. In the case of $n$-Si, for example, the following relationships take place:

$d n / d t=\left(1 / q \mu_{n}\right) \cdot d \sigma_{s}(t) / d t$,

$E_{s}=\left(1 / \varepsilon \varepsilon_{0}\right) \cdot \Delta \sigma_{s} / \mu_{n}$,

where $d n / d t$ is the change in the electron concentration in the c-band as a result of their transition from SES to cband, $q$ - electron charge, $\mu_{n}$ - electron mobility (in the first approximation, it corresponds to the bulk one), $\varepsilon-$ dielectric permittivity of $\mathrm{Si}, \varepsilon_{0}=8.85 \cdot 10^{-12} \mathrm{~F} / \mathrm{m}, \Delta \sigma_{s}-$ initial change in conductance after charging the capacitor, $E_{s}-$ the value of the electric field at Si surface [20, 21].

In accord with works $[51,52]$, the velocity $(d n / d t)$ of non-equilibrium depletion relaxation as a result of electron transitions from SES into c-band due to the thermal-and-field mechanisms can be written as:

$$
\begin{aligned}
& d n / d t=\kappa N_{t} C_{n} \exp \left(-E_{t} / k T+\gamma E_{s}{ }^{1 / 2}+v E_{s}{ }^{2}\right), \\
& \gamma=\alpha q^{3 / 2} / k T\left[1+\left(\left(E_{t}-B\right) k T\right) / \sigma^{2}\right], \\
& \nu=(1 / 24)\left((q \hbar)^{2} /\left(m(k T)^{3}\right)\left[1+\left(\left(E_{t}-B\right) k T / \sigma^{2}\right)\right]^{3},\right.
\end{aligned}
$$

where $N_{t}$ is the concentration of SES filled with electrons; $C_{n}-$ cross-section of capturing the electron by a surface center with the energy position $E_{t}$ relatively to c-band; $\kappa$ - coefficient that only weakly depends on $T$ and $E_{s} ; \sigma$ and $B$-parameters of the theory for multiphonon processes in the local center $E_{t} ; \alpha$ - dimensionless coefficient with the value dependent on the potential profile in the vicinity of the Coulomb center (in most cases $\alpha<1$ ). B parameter is associated with a shift from the equilibrium position of atoms that surround the local center $\left(E_{t}, N_{t}\right)$, when electron passes from the local center to the allowed band (c-band). Connected with this process is also $\sigma$ parameter that characterizes an effective coupling between this electron and at the $\left(E_{t}, N_{t}\right)$ center and ambient phonons. When temperatures exceed the Debay one, the relation $\sigma^{2}=4 B k T$ takes place $[51,52]$. It should be noted that the influence of electron-phonon interaction on ionization of the deep center in a strong electric field was also considered in [56].

The term $\exp \left(-E_{t} / k T\right)$ in the equation (3) characterizes thermal excitation of electrons from SES into c-band. This excitation can be accelerated due to a 
strong electric field via the following mechanisms: 1) field decreases the potential barrier for electron transitions from SES into c-band (if SES are of the Coulomb character) due to the Frenkel effect (term $\left.\exp \left(\gamma E_{s}^{1 / 2}\right)\right)$; 2) field accelerates electron transitions $E_{t} \rightarrow$ c-band due to the Franz-Keldysh effect (term $v E_{s}{ }^{2}$ ). In dependence of the values of the field $E_{s}$, temperature and parameters $\alpha, \sigma, B$, the term $\exp \left(v E_{s}{ }^{2}\right)$ will be dominant, if the following condition is valid:

$\alpha<(1 / 24)\left(q^{1 / 2} \hbar^{2} / m(k T)^{2}\right)\left[1+\left(E_{t}-B\right) k T / \sigma^{2}\right]^{2} E_{s}^{3 / 2}$.

\subsection{Relaxation of non-equilibrium depletion on atomically clean, real and thermally oxidized silicon surfaces}

Dependences $d n / d t\left(E_{s}^{2}\right), d p / d t\left(E_{s}^{2}\right)$ for $n-\mathrm{Si}$ and $p$-Si, respectively, were observed by us on atomically clean $\mathrm{Si}$ surfaces as well as on real and then thermally oxidized surfaces $[30,31,37]$. It means that the relations (6) are valid.

However, the physical and chemical state of these surfaces determines the number of peculiarities in relaxation of non-equilibrium depletion. In particular, when a real surface is aged and a relatively stable oxide layer $(\sim 20$ to $40 \AA)$ is created in air, tunnel transport mechanisms appear in relaxation of non-equilibrium depletion. Shown in Fig. 5 are the oscillograms with appearance of these mechanisms at $p$-Si surface $(\rho=$ $2300 \mathrm{Ohm} \cdot \mathrm{cm})$. When the voltage on the capacitor reaches $300-400 \mathrm{~V}$, relaxation of the non-equilibrium conductance is close to the exponential one [22] with decreasing the relaxation time $\tau$ within the range $2 \cdot 10^{3}$ to $6 \cdot 10^{-3} \mathrm{~s}$ under increasing the temperature from 180 up to $270 \mathrm{~K}$. The activation energy of the process determined from the dependence $\tau(1 / T)$ is equal to $0.48 \mathrm{eV}$ and corresponds to the energy position of SES, from which hole transitions into $\mathrm{v}$-band take place. In dependence of the temperature of measurements when the voltage on the field electrode of the capacitor is $V=800$ to $1400 \mathrm{~V}$,

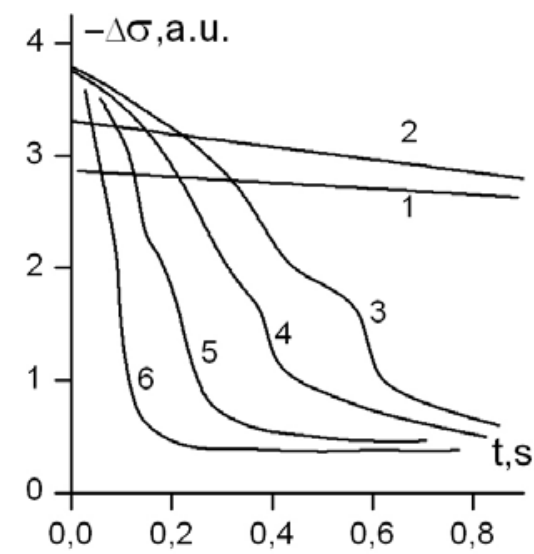

Fig. 5. Oscillograms of the non-equilibrium field effect for high $V_{\mathrm{M}}$ values on the field electrode $(1-1200,2-1400$, $3-1700,4-1800,5-1900,6-2000 \mathrm{~V})$ for the aged real surface of $p-\mathrm{Si}(2300 \mathrm{Ohm} \cdot \mathrm{cm})$ at $T=190 \mathrm{~K}$. one can observe acceleration of non-equilibrium depletion relaxation at the very beginning after switching $V$ on, while at the voltages $V=1300-1800 \mathrm{~V}$ we found additional parts of accelerated relaxation: at $T=220-250 \mathrm{~K}-$ one, and at $T=90-205 \mathrm{~K}-$ two for the times $t_{1}$ and $t_{2}$, respectively, after turning the voltage on $V(t=0)$. Typical oscillograms of these processes are shown in Fig. 5.

The dependence of the relaxation velocity $d p / d t$ at the origin of relaxation on $E_{\mathrm{s}}$ was determined using the formulae (1) to (5). It was ascertained that realized are the dependences $d p / d t\left(E_{s}^{2}\right)$ (Fig. 6). The activation energy determined from the dependences $d p / d t(1 / T)$ within the range $202-262 \mathrm{~K}$ at the fixed value $E_{s}=$ $3.3 \cdot 10^{4} \mathrm{~V} / \mathrm{cm}$ is equal to $0.48 \mathrm{eV}$, which coincides with the activation energy that was determined earlier from the dependences $\tau(1 / T)$ for those $V$ values when electric field acceleration of relaxation was not yet observed. It is indicative of the fact that in both cases we deal with hole transitions into v-band from the same SES with the energy position $E_{t}=0.48 \mathrm{~V}$ relatively to $\mathrm{v}$-band, but at high $V$ values these transitions are accelerated by $E_{s}$ due to the Franz-Keldysh effect. Coincidence between $E_{t}$ values from the dependences $\operatorname{dp} / d t\left(T^{-1}\right)$ and $\tau\left(T^{-1}\right)$ confirms apparent independence of the coefficient $v$ values from the formulae (3) to (5) of temperature, that is the condition $\left(E_{t}-B\right) k T / \sigma^{2}>1$ is valid. This condition should be valid for all the temperatures of our investigations $(90-270 \mathrm{~K})$, as in the opposite case, as it follows from calculations, the determined $E_{\mathrm{s}}$ values cannot provide the observed acceleration of relaxation. Bearing in the mind these $E_{\mathrm{s}}$ values corresponding to the origin of relaxation acceleration and taking into account the condition $E_{t}>>B$ (as it was done in [51]), one can estimate the value of $\sigma$ parameter. It turned out that this value does not exceed $0.04 \mathrm{eV}$.

Adduced in Figs 7 and 8 in the semi-logarithmical scale are, respectively, dependences for times $t_{1}$ and $t_{2}$ on $V$ for temperatures characteristic of appearance of

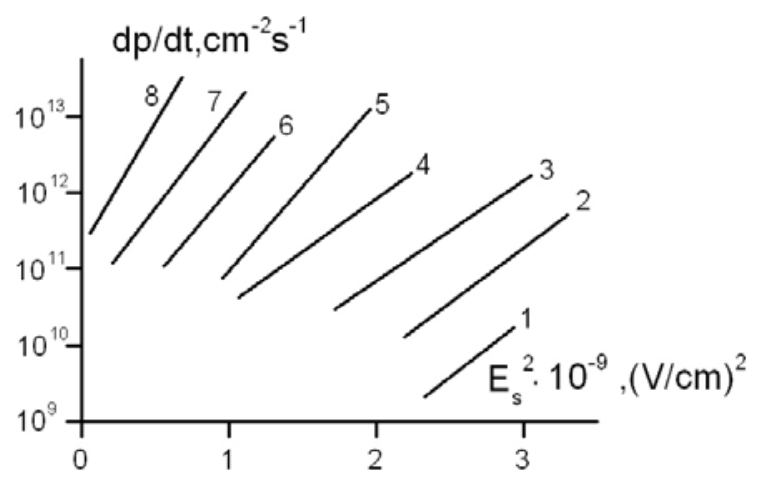

Fig. 6. Dependences of the relaxation velocity for nonequilibrium depletion in the field effect on the aged real surface of $p$-Si on the field $E_{s}$ at the silicon surface $(T, \mathrm{~K}$ : $1-130,2-152,3-166,4-190,5-202,6-223$, $7-240,8-262)$. 
additional parts inherent to accelerated relaxation. It is seen that the times $t_{1}$ and $t_{2}$ that change within the range $4 \cdot 10^{2}$ to $1 \cdot 10^{-2} \mathrm{~s}$ grow exponentially with $V$ and, besides, they drop exponentially with the increasing temperature. In our opinion, the appearance of these additional parts of accelerated relaxation is caused by the voltage $V$ redistribution in the relaxation process between those its fraction that drop in semiconductor $V_{s}$ and in the dielectric spacing $V_{d}$. Due to the change in SES charge, $V_{s}$ value decreases with time, while $V_{d}$ increases. The voltage $V_{d}$ drops in mica $\left(V_{c}\right)$ and in the layer of the real silicon oxide $\left(V_{\mathrm{ox}}\right)$. Under the conditions of our experiment, the following relations take place:

$V_{\mathrm{ox}}=\left[\varepsilon_{c} d_{\mathrm{ox}} /\left(\varepsilon_{\mathrm{ox}} d_{c}\right)\right]\left(V_{d}-V_{\mathrm{ox}}\right) \approx\left[\varepsilon_{c} d_{\mathrm{ox}} /\left(\varepsilon_{\mathrm{ox}} d_{c}\right)\right] V_{d}$,

where $\varepsilon_{\mathrm{c}}, d_{c}, \varepsilon_{\mathrm{ox}}, d_{\mathrm{ox}}$ are dielectrical permittivities and thicknesses of mica and Si oxide layer. Therefore, the growth of the $V_{d}$ value with time in the course of relaxation results in proportional growth of $V_{\text {ox }}$, which promotes tunnel transitions of holes from SES, that are present in oxide or on its external surface, into the silicon v-band. This can explain the appearance of the additional parts of accelerated relaxation.

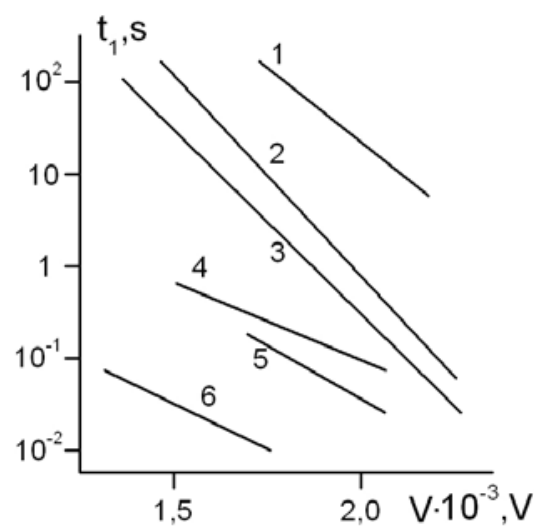

Fig. 7. Dependences on the field electrode voltage value for the time $t_{1}$, that characterizes appearance of the first additional acceleration of non-equilibrium depletion relaxation for the aged surface of $p$-Si (T, K: 1 - 99, 2 - 130, 3 - 166, 4 - 190, $5-202,6-240)$.

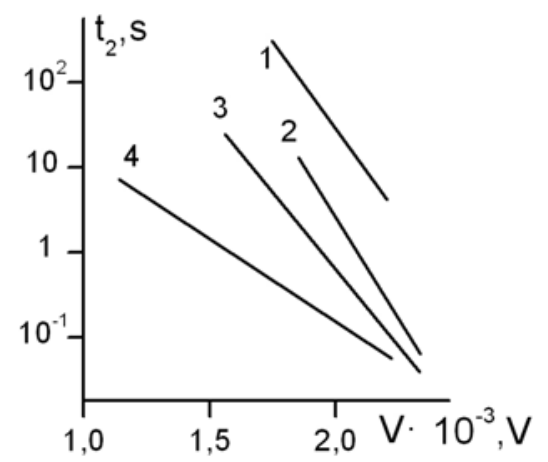

Fig. 8. Dependences on the field electrode voltage value for the time $t_{2}$, that characterizes appearance of the second additional acceleration of non-equilibrium depletion relaxation for the aged surface of $p-\mathrm{Si}(T, \mathrm{~K}: 1-99,2-130,3-166,4-202)$.
$V_{s}$ value in every time moment can be determined as: $V_{s}(t)=\left[q \rho_{0} /\left(2 \varepsilon \varepsilon_{0}\right)\right] d^{2}{ }_{\text {dep }}(t)=$

$=\left(q \rho_{0} d^{2}{ }_{\mathrm{Si}} /\left(2 \varepsilon \varepsilon_{0}\right)\left[V_{r}(t) / V_{r 0}\right]^{2}\right.$,

where $\rho_{0}$ is a specific concentration of holes in $\mathrm{Si} ; d_{\mathrm{Si}}$, $d_{\text {dep }}$ - thicknesses of the silicon sample and its depleted layer, respectively; $V_{r 0}$ - initial voltage $(t=0)$ on the resistance connected into circuit in series with the sample of $\mathrm{Si}$; $V_{r}(t)$ - change of voltage on the resistance after applying the external voltage $V$ to the capacitor.

$V_{s}(t)$ values that were determined in the moment $t_{2}$, when the second part of accelerated relaxation begins, turned out to be close for all $V$ values at a definite $T$, that is the relaxation acceleration takes place due to growing $V_{d}$ Shown in Fig. 9 are the dependences $d p_{2} / d t$ at the time moment $t_{2}$ on $V_{d}$. It is seen that exponential growth of $d p_{2} / d t$ with increasing the value $V_{d} \sim V_{\text {ox }}$ takes place, which agrees with the conception of tunneling charge carriers through the oxide layer into semiconductor [12]. Also, Fig. 9 confirms that $d p_{2} / d t$ grows with $T$. The activation energy determined using the dependence $d p_{2} / d t\left(T^{-1}\right)$ is equal to $0.25 \mathrm{eV}$. Thus, the process of relaxation acceleration at $t_{2}$ possesses a tunnel-activation character. Transition of holes into Si v-band from SES of oxide can be realized by two ways: 1) holes tunnel to SES at the boundary silicon - oxide and then are thermally excited into $\mathrm{Si}$ v-band; 2) holes first are thermally excited onto the levels in oxide that are against $\mathrm{Si}$ v-band, and then do the tunnel transition. As seen from calculations, the electric field in the oxide layer is sufficient to draw up the levels filled with holes by the energy distance $0.25 \mathrm{eV}$ above the v-band.

Acceleration of relaxation at the moment $t_{1}$ is also of the tunnel-activation nature. However, in this case acceleration appears at lower fields in oxide than that at the moment $t_{2}$, therefore, tunneling should be facilitated due to more close location of respective SES (traps) to the interface silicon - oxide. The fact that traps, from which holes tunnel at the moment $t_{2}$, are located far from the interface can be confirmed by data of processing the samples in weakly concentrated HF that lowers the thickness of the oxide layer and results in disappearance of the second part $\left(t_{2}\right)$ in accelerated relaxation.

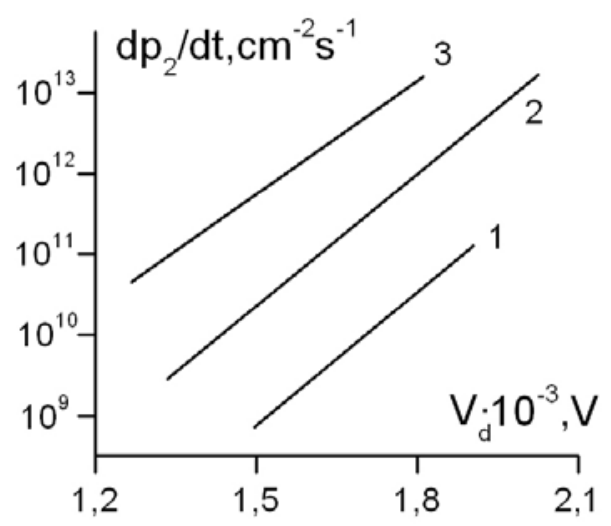

Fig. 9. Dependences of the relaxation velocity $d p_{2} / d t$ at the moment $t_{2}$ on the voltage that drops in the dielectric spacing $V_{d}$ $(T, \mathrm{~K}: 1-142,2-166,3-202)$. 
Acceleration of relaxation at the moment $t_{1}$ is also characterized by the fact that it takes place at rather high fields in semiconductor $E_{s}$, thereof, relaxation can be accelerated by both the voltages $V_{d}$ and $V_{s}$. Indeed, the values $d p_{1} / d t$ at the moment $t_{1}$ grow due to both these voltages, which is indicative of validity of the tunnelactivation mechanism that includes the Franz-Keldysh effect as well. It means that tunneling is realized via virtual over-band states that are caused by this effect. However, it should be noted that within the range $T=$ 200-240 K with increasing the depleting voltage $V$, the value $V_{d} \sim V_{\text {ox }}$ grew higher, while at $T<200 \mathrm{~K}$ when higher fields $E_{s}$ are realized at the surface of $p$-Si (Fig. 6) the change of $V$ at the moment $\mathrm{t}_{1}$ is accompanied by dominant changes in the $V_{s}$ value, and the value $V_{d}$ can be considered as independent of $V$ with a sufficient accuracy.

Depicted in Fig. 10 are the dependences of $d p_{1} / d t$ on the voltage $V_{s}$ for two temperatures. One can see that these dependences are exponential, and $\ln \left(d p_{1} / d t\right) \sim$ $V_{s} \sim E_{s}^{2}$ [20]. It means that the essential role in relaxation acceleration at the moment $t_{1}$ is played by tunneling to over-band states caused by the Franz-Keldysh effect.

The dependences $\Delta \sigma(t)$ enabled us to estimate the amount of holes that pass from SES (traps) located in oxide and at the interface $p$-Si - oxide into $p$-Si v-band. It turned out that at the initial moment of relaxation the number of hole transitions is changed with $T$ and $V$ to some extent and lies within the range $3 \cdot 10^{10}$ to $1.4 \cdot 10^{11} \mathrm{~cm}^{-2}$. The amount of holes tunneling from traps remote from the interface $\mathrm{Si}$ - oxide at the moment $t_{1}$ into $p$-Si v-band lies within the range $2 \cdot 10^{10}$ $1 \cdot 10^{11} \mathrm{~cm}^{-2}$, and at the moment $t_{2}$ (from the traps in oxide close to $\mathrm{Si}$ ) within the limits $7 \cdot 10^{10}-1.3 \cdot 10^{11} \mathrm{~cm}^{-2}$. These results confirm that in the process of depletion relaxation approximately the same contributions are given both by SES of Si-oxide interface and two groups of traps located in the oxide layer of $p$-Si.

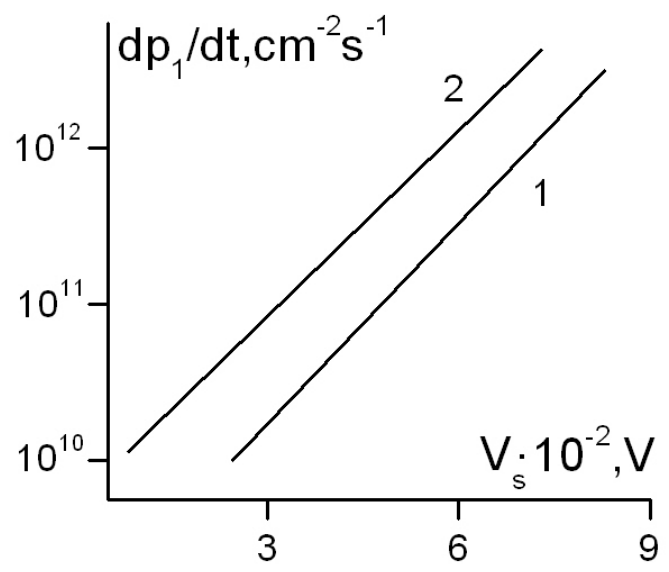

Fig. 10. Dependences of the relaxation velocity $d p_{1} / d t$ at the moment $t_{1}$ on the voltage drop in semiconductor $V_{s}(T, \mathrm{~K}$ : $1-152,2-190)$.
The majority carriers (holes) that pass into $p$-Si vband from SES (traps) due to thermal and field mechanisms under a further increase in the voltage $V$ can be heated in SCR and result in appearance of an avalanche as a consequence of impact ionization causing nativity of electron-hole pairs $[49,50]$. In this case, the impact ionization is realized via interband $(\mathrm{v} \rightarrow \mathrm{c})$ electron transitions, except heavily doped samples.

As seen from analysis, the Franz-Keldysh effect is the reason for non-equilibrium depletion relaxation acceleration at high $V$ on atomically clean surfaces of $n$ - and $p$-Si [21]. In these cases, the absence of Si layer oxide enables to observe the acceleration of depletion relaxation in high $V$ only due to transitions of charge carriers between SES of the atomically clean surface and respective bands of majority carriers in $\mathrm{Si}$. At the same time, contribution of oxide traps into relaxation acceleration is absent. Fig. 11 shows the dependences $d p / d t\left(E_{s}^{2}\right)$ and $d n / d t\left(E_{s}^{2}\right)$ obtained in investigations of $p$-Si (line $1,101 \mathrm{~K}$ ) and $n$-Si (line $2,116 \mathrm{~K}$ ). Energy positions of SES on atomically clean surfaces that provide the dominant contribution to non-equilibrium depletion relaxation were obtained from temperature investigations of $d p / d t$ and $d n / d t$ values at moderate magnitudes of $V$. It turned out that their distance from the respective allowed bands is $0.4 \mathrm{eV}$. The calculated parameter of multiphonon processes on atomically clean surfaces $\sigma$ is equal to $0.023 \mathrm{eV}$ and has the least value in investigations of $n$ and $p$-Si samples with different physical-and-chemical states of the surface. It seems reasonable as SES possess ambient continuum on the atomically clean surfaces only from one side (Si side), and electron (hole) transitions are accompanied by "one-side" silicon phonons.

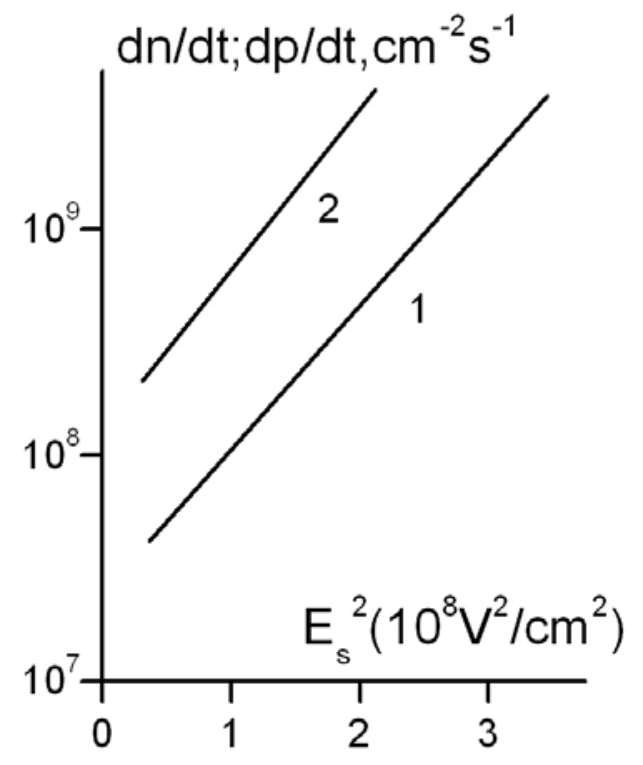

Fig. 11. Dependences of the relaxation velocity for nonequilibrium depletion on atomically-clean silicon surfaces $(1-p$-Si, $2300 \mathrm{Ohm} \cdot \mathrm{cm}, 101 \mathrm{~K} ; 2-n-\mathrm{Si}, 170 \mathrm{Ohm} \cdot \mathrm{cm}$, $116 \mathrm{~K})$. 
Influence of silicon surface thermal oxidation on the non-equilibrium depletion relaxation was investigated using the samples of $n$-Si $(180 \mathrm{Ohm} \cdot \mathrm{cm})$ [31]. Depicted in Figs. 12 and 13 are the dependences $d n / d t\left(E_{s}^{2}\right)$ obtained before and after surface oxidation. Comparison of them allows to draw a conclusion that for the similar $T$ and $E_{\mathrm{s}}$ the rate of relaxation $d n / d t$ on the thermally oxidized surface is the order of value less than that for the real surface. First of all, it is associated with lowering the concentration of BES (SES) after silicon oxidation. However, during the oxidation process, changed are other parameters, too, in particular $E_{t}$ and $\sigma$. The value $E_{t}$ for real and oxidized surfaces can be determined using the temperature dependences of $d n / d t$ at a definite value of $E_{s}$. Fig. 14 demonstrates these dependences for the value $E_{s}=1.4 \cdot 10^{4} \mathrm{~V} / \mathrm{cm}$. $E_{t}$ values determined from the dependence $d n / d t\left(T^{-1}\right)$ at $T=230 \mathrm{~K}$ for real and oxidized surfaces are 0.48 and $0.63 \mathrm{eV}$, respectively. Thus, BES (SES) from which electrons pass to c-band are located at larger energy distance from c-band after silicon oxidation than that for the real surface. It is not expected that transition of electrons from BES inherent to oxidized surface is a part of the process providing generation of electron-hole pairs with participation of these BES. When $T<230 \mathrm{~K}$, the slope of $d n / d t\left(T^{-1}\right)$ dependences (Fig. 14) for these surfaces is decreased. It can be caused by both changes of BES (SES), from which electrons are emitted into c-band, and manifestation of the Frenkel effect at low temperatures $[52,53]$.

Taking into account that the inequality $E_{t}>>B$ is valid, as a rule, in these conditions $[51,52]$, the parameter of electro-phonon coupling $\sigma$ can be determined for every $T$ by using the equation:

$\sigma=\left(\hbar^{2} / 24 m\right)^{1 / 6} E_{t}^{1 / 2}\left\{\Delta\left(q^{2} E_{s}^{2}\right) / \Delta[\ln (d n / d t)]\right\}^{1 / 6}$.

Presented in Fig. 15 are the dependences $\sigma(T)$ for real (1) and thermally oxidized (2) surfaces. For all the temperatures, the $\sigma$ value inherent to the thermally oxidized surface with the layer of thermal $\mathrm{SiO}_{2}(d \sim$ $1000 \AA$ ) is larger than that for the real surface with the

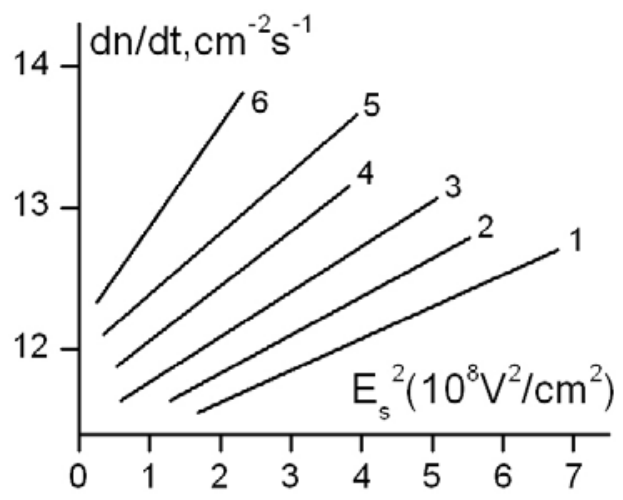

Fig. 12. Dependences of the relaxation velocity for nonequilibrium depletion $d n / d t$ on real $n$-Si surface on the electric field value $E_{s}$ on the surface at $T, \mathrm{~K}: 1-204,2-$ $216,3-232,4-242,5-255,6-268$. layer of natural oxide $(d \sim 30 \AA)$. It is indicative of a preferential role of phonons present in the oxide thermal layer as compared with phonons in the layer of natural oxide, which can be associated with more elastic structure of the former in comparison with a relatively friable structure of natural oxide. Rearrangement of local center $\left(E_{t}, N_{t}\right)$ environment, which accompanies electron transition from this center to c-band, is related with phonon excitation and needs larger energy expenses in the case of thermal oxide [56].

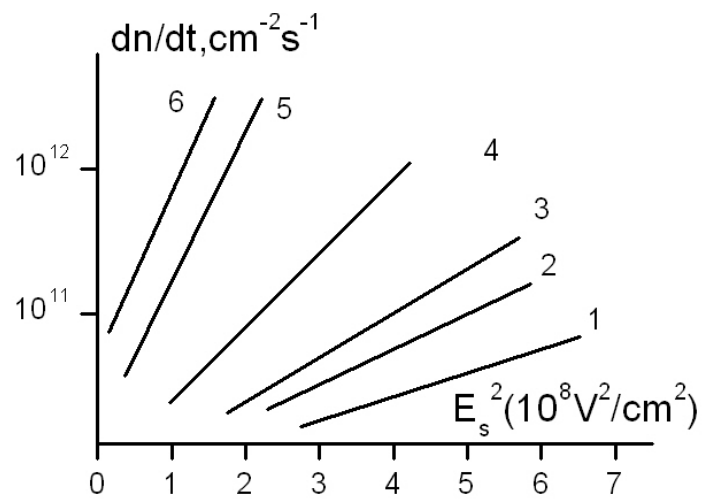

Fig. 13. Dependences $d n / d t\left(E_{s}^{2}\right)$ after thermal oxidation of $n$ Si at $T, K: 1-210,2-221,3-233,4-247,5-261,6-272$.

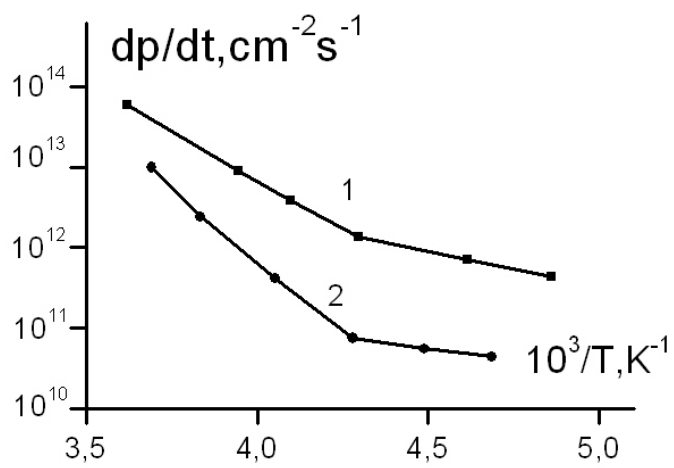

Fig. 14. Dependences $d n / d t$ on $1 / T(1-$ real $n$-Si surface, 2 - thermally oxidized surface).

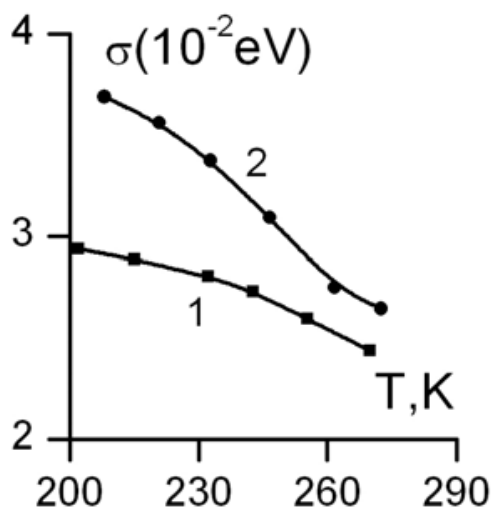

Fig. 15. Dependences of the parameter $\sigma$ value for electronphonon coupling on the temperature of real (1) as well as thermally oxidized (2) $n$-Si surface. 
3.2. Non-equilibrium depletion relaxation in strong electric fields on a real silicon surface doped with metals

As it was noted above, to find possible mechanisms for acceleration of non-equilibrium depletion effect relaxation in strong electric fields, it is necessary to change a physical-and-chemical state of silicon surface. In many works generalized in the monograph [15], we showed that treatment of samples in metal salt water solutions results in deposition of metal ions on the surface. In this case, discharging the metal ions with higher positive electrochemical potential than that of silicon causes creation of thin-dispersion island-like metal films, parameters of which depend on the state of silicon surface during ion deposition, their nature and concentration in the solution, $\mathrm{pH}$ of the latter, time of deposition, etc. It was presumed earlier that adsorption of metal ions with a higher negative electrochemical potential than that of silicon is realized via substitution of protons in $\mathrm{OH}$ groups (covering the surface [16]) by these ions. Therefore, the concentration of adsorbed ions should not exceed that in monolayer. However, in a number of our works also summarized in [15] we showed that adsorption of ions of the so-called electronegative metals can provide coatings with the thickness considerably exceeding that of monolayers. Moreover, instead of an amorphous silicon oxide layer we deal with a metal silicate structure adsorbed that can have a polycrystalline character, and its thickness can be higher than that of the initial oxide layer.

We investigated acceleration of non-equilibrium depletion relaxation in strong fields by using the samples of silicon $(p-\mathrm{Si}, \rho=2300 \mathrm{Ohm} \cdot \mathrm{cm})$, the real surface of which was doped with $\mathrm{Zn}[33,37]$ (electronegative metal) as well as with $\mathrm{Au}[32,35,37]$ (electropositive metal).

Doping with $\mathrm{Zn}$ was performed in two ways: 1) treatment of silicon samples in $1 \% \mathrm{ZnCl}_{2}$ water solution with $\mathrm{pH}=6$ for 30 min (way 1);2) treatment of silicon samples in $\mathrm{ZnCl}_{2}$ solution with $\mathrm{pH}=9$ for $20 \mathrm{~min}$ (way 2). Before doping, in both cases Si samples were kept for $30 \mathrm{~min}$ in boiling doubly distilled water to improve solvability of $\mathrm{Si}$ oxide layer and further creation of polycrystalline silicate layers, growth of which was controlled using ellipsometer and electronograph. In the first way, we obtained a silicate layer $(d=30 \AA)$ that consist of the mixture $2 \mathrm{ZnO} \cdot \mathrm{SiO}_{2}$ and $\mathrm{Zn}(\mathrm{OH})_{2} \cdot \mathrm{Si}_{2} \mathrm{O}_{7} \cdot \mathrm{H}_{2} \mathrm{O}$; while in the second way we obtained the silicate layer $(d=60 \AA)$ mainly of $\mathrm{Zn}(\mathrm{OH})_{2} \cdot \mathrm{Si}_{2} \mathrm{O}_{7} \cdot \mathrm{H}_{2} \mathrm{O}$ composition.

Our analysis of non-equilibrium conductance relaxation in strong electric fields at the initial moment of their turning on for the samples non-doped with $\mathrm{Zn}$ ( $p$-Si after boiling in water) and doped with $\mathrm{Zn}$ by using the way 1 indicates that the dependences of $d p / d t \sim \exp \left(v E_{s}^{2}\right)$ type are realized. In this case, the value $d p / d t$ for the surface doped with $\mathrm{Zn}$ is two orders less than for the non-doped sample at the same values of $T$ and $E_{s}$. Besides, within the temperature range $240-140 \mathrm{~K}$ for the surface doped with $\mathrm{Zn}$, acceleration of relaxation by electric field begins at higher values of $E_{s}\left((2.5-4.5) \cdot 10^{4} \mathrm{~V} / \mathrm{cm}\right)$ and less sharp than for the boiled surface of $p$-Si $E_{s} \approx(0.6-$ $2.1) \cdot 10^{4} \mathrm{~V} / \mathrm{cm}$. All this is in good agreement with the results of comprehensive investigations of $\mathrm{Si}$ surface doped with $\mathrm{Zn}$, where the authors showed, first of all, an essential decrease in the SES concentration $N_{t}$ after doping with $\mathrm{Zn}[15]$.

Doping $p$-Si with $\mathrm{Zn}$ by using the way 2 results in some higher values of $d p / d t$ for the same $T$ and $E_{s}$ than doping in the way 1 . Besides, at lower $E_{s}$ one can observe acceleration of relaxation by the field $E_{s}$, which is caused by the Frenkel effect $[52,53]$. Depicted in Figs. 16 and 17 are the dependences $d p / d t$ on $E_{s}$ for two ways of doping $p$-Si with $\mathrm{Zn}$. If using the way 2, acceleration of relaxation by the field $E_{s}$ is already observed at $E_{s}=(0.5-6) \cdot 10^{3} \mathrm{~V} / \mathrm{cm}$.

Fig. 18 shows the temperature dependences for the coefficients $v$ and $\gamma$ (see formulae (4) and (5)) that were calculated in accord with slopes inherent to the dependences $\ln d p / d t\left(E_{s}^{2}\right)$ and $\ln d p / d t\left(E_{s}{ }^{1 / 2}\right)$. It is seen that the values of $v$ are several times decreased after doping. The values of $v$ and $\gamma$ coefficients grow with temperature (Fig. 18). In the case of the $p$-Si surface doped with $\mathrm{Zn}$ by using the way 2 , at $T>100 \mathrm{~K}$ and definite $E_{s}$ values one can observe a change from the dependence $d p / d t \sim \exp \left(\gamma E_{s}{ }^{1 / 2}\right)$ to the dependence $d p / d t \sim \exp \left(v E_{s}{ }^{2}\right)$. It takes place, if with growing $E_{s}$ the condition (6) becomes valid. Using the slopes of the dependences $d p / d t\left(T^{-1}\right)$, we calculated the respective activation energies. In the cases of "boiled" $p$-Si surface and that doped with $\mathrm{Zn}$ by using the way 1, the activation energy is equal to $0.46 \mathrm{eV}$, while for doped by using the way 2 it is equal to $0.14 \mathrm{eV}$. These values correspond to positions of $E_{t} \mathrm{SES}$, from which the hole emission into $\mathrm{v}$-band takes place.

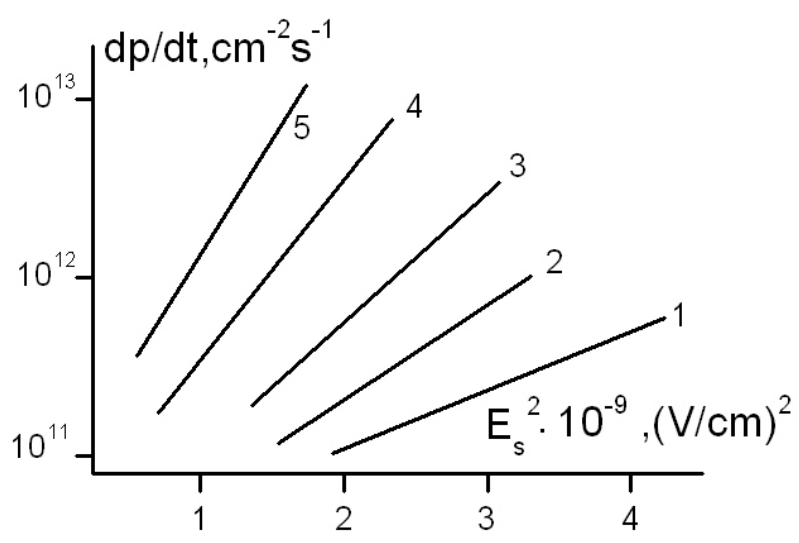

Fig. 16. Dependences of the relaxation velocity for nonequilibrium depletion $d p / d t$ on the field $E_{s}$ value on $p$-Si surface $(2300 \mathrm{Ohm} \cdot \mathrm{cm})$ after doping it with $\mathrm{Zn}$ by using the way $1(T, \mathrm{~K}: 1-158,2-208,4-221,5-239)$. 


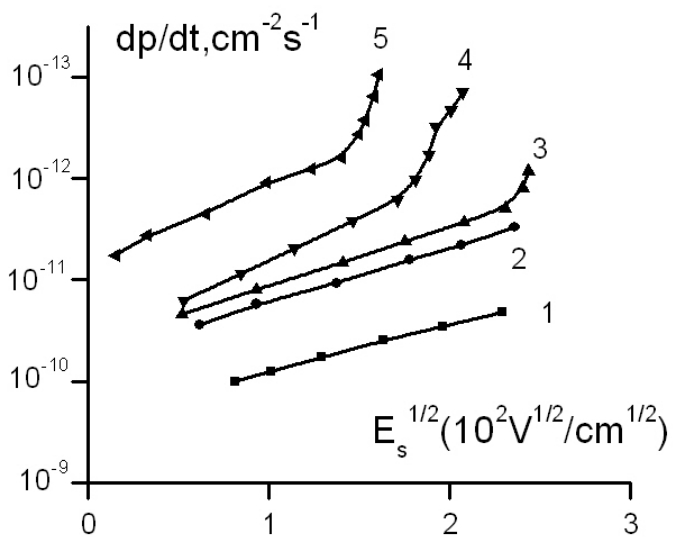

Fig. 17. Dependences of $d p / d t$ on the field $E_{s}$ value on $p$-Si surface after doping it with $\mathrm{Zn}$ by using the way 2 ( $T, \mathrm{~K}$ : $1-148,2-176,3-192,4-218,5-243)$.

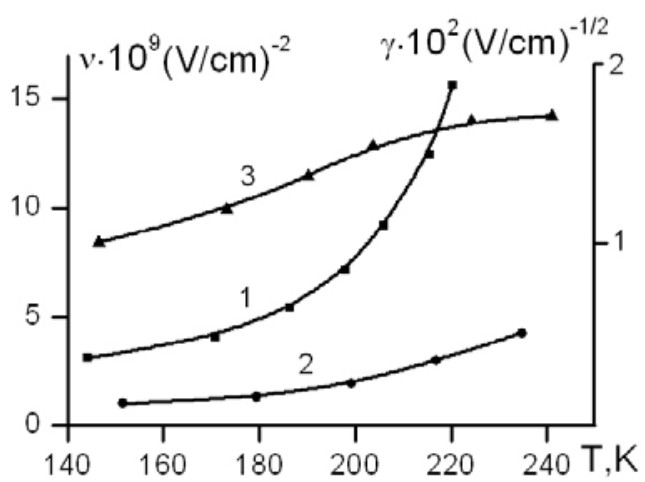

Fig. 18. Dependences of the coefficients $v(T)(1,2)$ and $\gamma(T)$ (3). 1 - real $p$-Si surface $\left(2300 \mathrm{Ohm}^{\circ} \mathrm{cm}\right)$ after boiling it in water. 2, 3- surfaces doped with $\mathrm{Zn}$ by using the ways 1 and 2 , respectively.

Knowing the values $E_{t}$ and $v$ as well as assuming $E_{t}>B[51]$, it is possible to determine the parameter $\sigma$ for definite $T$ values by using the formula (5). Fig. 19 presents dependences $\sigma(T)$. It turned out that within the temperature range 140 to $240 \mathrm{~K}$ for the boiled $p$-Si surface the $\sigma$ value changes within the interval 0.032 $0.024 \mathrm{eV}$, and for the doped with $\mathrm{Zn}$ (way 1) - in the interval $0.040-0.032 \mathrm{eV}$. To estimate the $\sigma$ value for the surface doped with $\mathrm{Zn}$ by using the way 2 is possible with account of the equality $\gamma E_{s}^{1 / 2}=v E_{s}^{2}$, when the rooted dependence of $d p / d t$ on $E_{s}$ is substituted with the quadratic one. At $218 \mathrm{~K}$, the value $\sigma \approx 0.032 \mathrm{eV}$. The dependence $\sigma$ on the type of $p$-Si surface layer indicates that charge carriers (holes) that are located in SES in the course of their emission into v-band interact not only with Si lattice phonons but with phonons of the surface layer covering $\mathrm{Si}$, too. The structure and composition of the surface layer that defines the phonon spectrum is essentially different in the samples under investigation. The oxide layer on the real surface after boiling it and respective creation of defects [57] has a friable structure. In this case, the electron-phonon coupling $(\sigma)$ will be relatively weak as compared with the case of $\mathrm{Zn}$ polysilicate film when $\sigma$ values are larger.

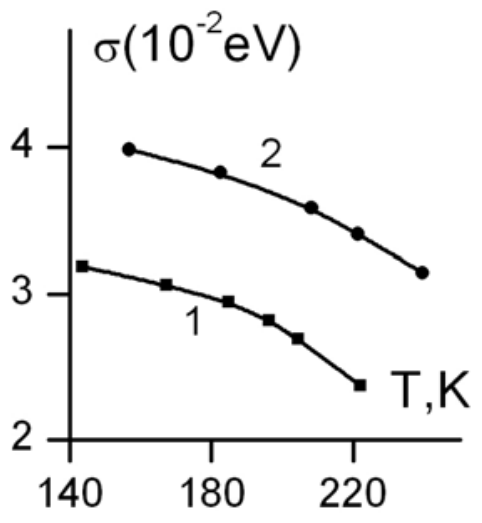

Fig. 19. Dependences of the electron-phonon coupling parameter $\sigma$ on temperature $(1-$ real $p$-Si surface, 2 - doped with $\mathrm{Zn}$ by the way 1 ).

It should be noted that for boiled and doped (way 1) $p$-Si surface we revealed an additional nonequilibrium depletion relaxation since some period after applying the voltage $V$ like that observed for the aged real surface. At $V=1100-1900 \mathrm{~V}$ within the temperature range $110-185 \mathrm{~K}$ for the boiled surface, we observed accelerated relaxation of $d p / d t$ values in $t_{1}=2.5 \cdot 10^{-2}$ $8 \cdot 10^{-1} \mathrm{~s}$ after switching $V$ on. The $d p / d t$ value grew exponentially with the voltage $V_{d}$ applied to the dielectric spacing, which is in proportion to the voltage drop in oxide $V_{\text {ox }}$. The activation energy value deduced from the dependence $d p / d t\left(T^{-1}\right)$ is $0.12 \mathrm{eV}$. This part of accelerated relaxation is caused by the tunnel-activation mechanism inherent to hole transitions from "slow" SES located in oxide into v-band.

The surface doped with $\mathrm{Zn}$ (way 1) is characterized by two parts of relaxation accelerated by field throughout the times $t_{1}$ and $t_{2}$ after switching $V$ on. The first part is observed after $t_{1}=10^{-2}-50 \mathrm{~s}$ at $110-240 \mathrm{~K}$ and $V=1100-2000 \mathrm{~V}$. The second part - throughout $10^{-1}$ $10 \mathrm{~s}$ at $110-210 \mathrm{~K}$ and $V=1300-2000 \mathrm{~V}$. The times $t_{1}$ and $t_{2}$ decreased exponentially with the growing $V$ and decreasing $T^{-1}$. The $d p / d t$ value grows exponentially with the voltage applied to semiconductor $V_{s}$ that is in proportion to $E_{s}{ }^{2}$ at the moment $t_{1}$, with the activation energy being $0.27 \mathrm{eV}$, as deduced from the dependence $d p / d t\left(T^{-1}\right)$. Thus, the relaxation acceleration at the moment $t_{1}$ is caused by the tunnel-activation mechanism of hole transitions from SES located in the surface silicate film into $\mathrm{Si}$ v-band, when the Franz-Keldysh effect still plays a significant role in field acceleration of relaxation. And conversely, at the moment $t_{2}$, the value $d p / d t$ grows exponentially with $V_{d}$ and does not practically depend on $V_{s}$. In this case, the tunnelactivation mechanism of relaxation acceleration (when the activation energy is less than $0.12 \mathrm{eV}$ ) is mainly caused by the value $V_{\text {ox }}$, as $V_{s}$ is relatively small.

On Si surface doped with $\mathrm{Zn}$ by using the way 2 , additional parts of accelerated relaxation at $t \geq 0$ were not revealed. It can be associated with location of slow 
SES inside the silicate film far from Si surface and a difference between their parameters as well as with lower values of electric fields both in $\mathrm{Si}$ and in the silicate film.

Let us proceed to consideration of non-equilibrium depletion relaxation accelerated by field when $p$-Si surface was doped with $\mathrm{Au}$ taken as an electropositive metal that forms island-like metal films on Si surface $[32,35]$. Doping $p$-Si with $\mathrm{Au}$ was performed treating the samples in $48 \%$ HF solution where $\mathrm{AuCl}_{3}$ salt concentration was changed within the range $5 \cdot 10^{-7}$ to $1 \cdot 10^{-4}$ mole/l. Relaxation of non-equilibrium depletion was investigated as earlier in the course of changing the sample conductance after applying the external voltage $V$. It was ascertained that the $d p / d t$ relaxation velocity at the initial moment $(t \geq 0)$ depends both on the temperature of measurements and, starting with some values, on the field value $E_{s}$, as well as on the concentration of the doping $\mathrm{Au}$ impurity. On the $p-\mathrm{Si}$ surface treated in $\mathrm{HF}$ solution not containing the $\mathrm{Au}$ impurity and with this impurity $\left(C=5 \cdot 10^{-7}\right.$ mole/l (at $T>120 \mathrm{~K})$ ), the dependence $d p / d t \sim \exp \left(v E_{s}{ }^{2}\right)$ takes place. However, the samples doped with higher $\mathrm{Au}$ concentrations indicated the relation $d p / d t \sim \exp \left(\gamma E_{s}^{1 / 2}\right)$ at $T \leq 170 \mathrm{~K}$. Considering various states of the surface in the following sequence real - treated with $48 \% \mathrm{HF}-$ doped $\left(5 \cdot 10^{-7} \mathrm{~mole} / \mathrm{l}\right)$, we found that the $d p / d t$ value grow by almost five orders. This essential growth of $d p / d t$ values for HF-treated and doped with Au surface is caused by several reasons and, first of all, by growth of the concentration of SES traps $N_{t}$ [58] as well as by increasing the capture cross-section $C_{p}$. The latter value was determined using extrapolation of dependences $\ln \tau\left(T^{-1}\right)$ at $T^{-1} \rightarrow 0[22,23]$, where $\tau$ is the time of nonequilibrium depletion relaxation under low (nonaccelerating) $V$ values. It turned out that for real (oxidized) surface $C_{p}=2 \cdot 10^{-15} \mathrm{~cm}^{2}$, for HF-treated $C_{p}=$ $1.5 \cdot 10^{-14} \mathrm{~cm}^{2}$, and for Au-doped $C_{p}=1 \cdot 10^{-12} \mathrm{~cm}^{2}$. The calculated $\sigma$ value for the Au-doped surface is $0.023 \mathrm{eV}$. When calculating the $\sigma$ value, we took into account that $E_{t}=0.45 \mathrm{eV}$ (as seen from the dependence $d p / d t\left(T^{-1}\right)$ within the temperature range 190-240 K) and $E_{t}>>B$.

From the dependences $d p / d t \sim \exp \left(\gamma E_{s}^{1 / 2}\right)$ that takes place at the concentration of doping Au impurity $C>1 \cdot 10^{-6}$ mole/l and their dependences $d p / d t\left(T^{-1}\right)$, we deduced the parameters corresponding to realization of the Frenkel effect promoting acceleration of relaxation. We found that $E_{t}=0.37 \mathrm{eV}, \gamma=6.7 \cdot 10^{-2}(300 / T)(\mathrm{V} / \mathrm{cm})^{-1 / 2}$. Then the condition $\sigma^{2} /\left(E_{t}-B\right)>k T$ for $E_{t}>>B$ is valid within the temperature range under investigation at $\sigma>0.07 \mathrm{eV}$. These $\sigma$ values allows to neglect the term $\exp \left(v E_{\mathrm{s}}^{2}\right)$ in the formula (3), and the field dependence of $d p / d t$ is determined by the term $\exp \left(\gamma E_{s}{ }^{1 / 2}\right)$. Therefore, transfer from quadratic dependences on $E_{s}$ to the rooted ones with growing $C$ is determined by the growth of the $\sigma$ parameter.

The results of the work [32] were reconsidered in [35] with account of non-homogeneity in $E_{s}$ values, which is caused by an island-like structure of the metal film. Presented in Fig. 20 are the calculated model and dependences $d p / d t\left(E_{s}^{2}\right)$ before and after introducing this correction.

It was revealed using electron-microscopic investigations [15] that when the $\mathrm{Au}$ concentration in solution reaches $5 \cdot 10^{-7}$ mole/l, created on the $p$-Si surface is an island-like $\mathrm{Au}$ structure with island dimensions $l_{i}=2 \cdot 10^{-7} \mathrm{~cm}$ and their concentration $\sim 1 \cdot 10^{11} \mathrm{~cm}^{-2}$ (distances between islands $L \approx 3 \cdot 10^{-6} \mathrm{~m}$; $l_{i}<<L$ ). After doping, the $p$-Si sample is oxidized in air, and the islands appear to be included into the film of real $\mathrm{Si}$ oxide, with the thickness being of the order of the island height $\Delta x$. The thickness of dielectric (mica) is $x_{0}$, and the thickness of the non-equilibrium depletion layer in $p$-Si considerably exceeds the $L$ value.

When applying the depleting voltage $V$ to this MDS structure, the $p$-Si surface potential under islands will be always higher (with account of the equipotential character of the field on the surface of each island) than that in some distance from the island that exceeds several times the island dimension $l_{i}$.
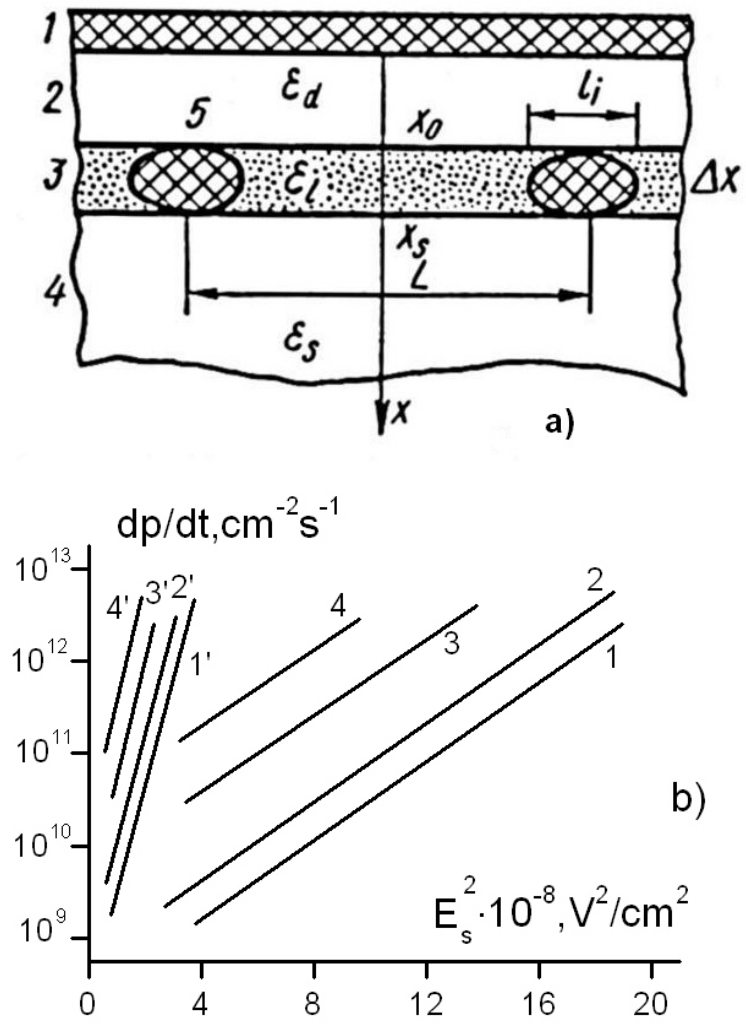

Fig. 20. a) MDS system: 1 - metal, 2 - dielectric, 3 - surface oxide film on real $p$-Si surface, 4 - semiconductor, 5 - islandlike metal inclusions (Au). b) Dependences of the relaxation velocity for the non-equilibrium field effect $(d p / d t)$ on the doped with $\mathrm{Au}$ real $p$-Si surface $(2300 \mathrm{Ohm} \cdot \mathrm{cm})$ on the field $E_{s}$ value on the $p$-Si surface without taking into account the additional field $\Delta E_{s}$ under islands of $\mathrm{Au}\left(1^{\prime}-4^{\prime}\right)$ as well as with account for $\Delta E_{s}(1-4)$ 
In this case, electric field lines of force are concentrated around the island, and the electric field $E_{s}$ on $p$-Si surface under the island takes a value by $\Delta E_{s}$ higher than the field $E_{s 0}$ that can be determined via $p$-Si conductance changes without account of non-homogeneity. Thus, to obtain correct dependences $d p / d t\left(E_{s}\right)$ one should find a value of the additional electric field under islands $\Delta E_{s}$. This value stems from the solution of 3D Poisson equation with $L>>l_{i}$, if one calculates first in a linear approximation the additional potential under islands $\Delta \varphi(x, r)$ and then $\Delta E_{s}$ :

$\Delta E_{s}=-d \Delta \varphi / d x=\left(4 \varepsilon_{s} \varepsilon_{d} E_{s 0} / \pi \varepsilon_{1}\left(\varepsilon_{s}+\varepsilon_{d}\right)\right)\left(\Delta x / l_{i}\right) R$,

where $t$ is $2 \mathrm{D}$ vector in the surface plane $(y, z) ; \varepsilon_{s}, \varepsilon_{d}, \varepsilon_{1}-$ values of dielectric permittivity for semiconductor, mica and oxide layer, respectively; $R$ - coefficient calculated with a computer and equal to 1.62-1.74 in dependence on the ratio $L$ to $l_{i}$. Calculations using the formula (10) result in

$\Delta E_{s}=1.3 E_{s 0} ; \quad E_{s}=E_{s 0}+\Delta E_{s}=2.3 E_{s 0}$.

Fig. 20b shows the dependences $d p / d t\left(E_{s}^{2}\right)$ : lines 1 to 4 were obtained with account of the correction $\Delta E_{s}, 1$ to $4^{\prime}$ - without account of this correction. It is seen that account of the correction results in a considerably less slope in the $d p / d t\left(E_{s}^{2}\right)$ dependences, which has the same magnitude for all $T$ values. It means that after introduction of this correction, the value of electronphonon coupling parameter (independent of $T$ ) grow:

$\sigma=(\hbar / 24 m)^{1 / 6} E_{t}^{1 / 2}\left[\Delta\left(e^{2} E_{s}^{2}\right) / \Delta(\ln (d p / d t))\right]$,

where $e, m$ are the charge and mass of holes, $E_{t}$ - energy position of SES. The $E_{t}$ value does not depend on the correction and is equal to $0.45 \mathrm{eV}$. Calculation of $\sigma$ with the correction $\Delta E_{s}$ results in $\sigma=0.035 \mathrm{eV}$ (without the correction $\sigma=0.023 \mathrm{eV}$ ), which is higher than the $\sigma$ value for the real surface $(0.028-0.031 \mathrm{eV})$. It means that excitation of holes from SES into v-band is essentially promoted by phonons not only of Si and oxide film on it but by phonons of the metal islands as well.

With the Au concentration $C \geq 1 \cdot 10^{-6}$ mole/l, when a considerable fraction of $p$-Si surface is covered with $\mathrm{Au}$, and the dependences $d p / d t \sim \exp \left(\gamma E_{s}{ }^{1 / 2}\right)$ are valid, $\Delta E_{s}$ correction can be not taken into account without noticeable errors in results. Given in Fig. 21 are the dependences of $d p / d t$ at the same value $E_{s}=$ $1.5 \cdot 10^{4} \mathrm{~V} / \mathrm{cm}$ and $d p / d t$ for the same $T=90 \mathrm{~K}$ on the concentration $C$ of the doping Au impurity. All of them have the $N$-like character. A similar shape of dependences on $C$ was earlier observed for the SES concentration and the velocity of surface recombination for non-equilibrium carriers after their generation by light. This $N$-similarity is caused by processes of creation and break of $\mathrm{Au}-\mathrm{Si}$ bonds in the course of nucleation and coagulation of islands [59, 60].
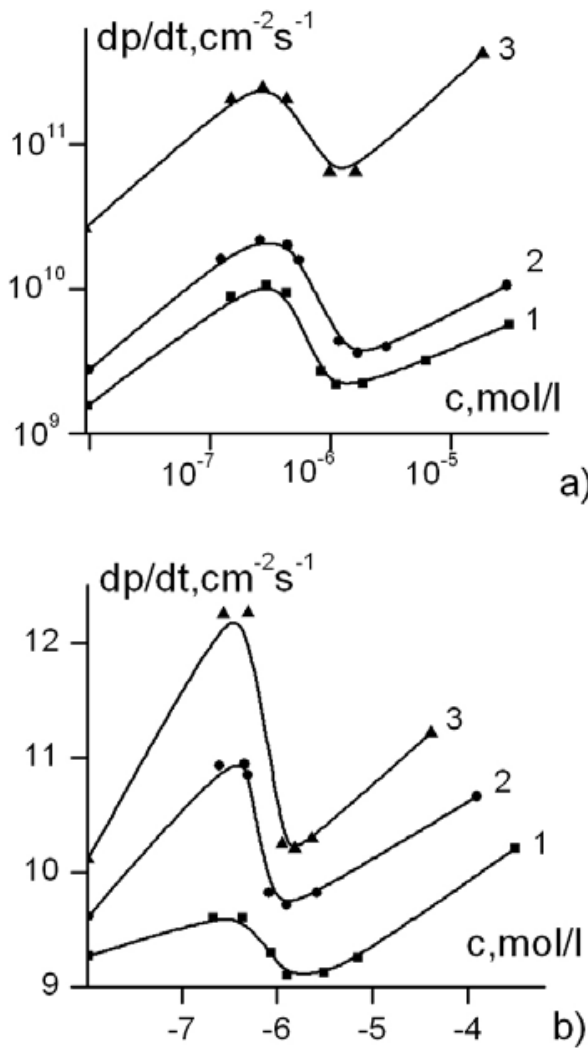

Fig. 21. Dependences of $d p / d t$ typical for the $p$-Si surface doped with $\mathrm{Au}$ on the concentration $C$ of the $\mathrm{Au}$ doping impurity in water solution: (a) - at $T, \mathrm{~K}: 1-84,2-127,3-$ $163 ; E_{s}=1.5 \cdot 10^{4} \mathrm{~V} / \mathrm{cm} ;(\mathrm{b})-$ at $E_{s}, \mathrm{~V} / \mathrm{cm}: 1-1 \cdot 10^{4}, 2-2 \cdot 10^{4}$, $3-3 \cdot 10^{4} ; T=90 \mathrm{~K}$

\section{Relaxation of non-equilibrium depletion in strong electric fields in MDS structures $\left(\mathrm{M}-\mathrm{SiO}_{2}-\mathrm{n}-\mathrm{Si}\right)$}

Acceleration of non-equilibrium depletion relaxation in strong electric fields considered in previous chapters was studied via measurements of the silicon samples conductance. It was also interesting to observe this phenomenon of acceleration directly in industrial structures metal-dielectric $\left(\mathrm{SiO}_{2}\right)$-semiconductor(silicon) (that is MDS structures) the most widely investigated abroad $[8,12]$. In this case, it is reasonable to measure the capacitance $C(t)$ of MDS structures after applying the voltage $V$ to them, which depletes silicon with majority charge carriers.

These investigations were performed in our works $[34,36]$. MDS structures were made using the wafers (100) of $n$-Si (KЕФ-4) thermally covered with the oxide layer ( $d=1200 \AA$ ) with use of "chlorine" technology to decrease the amount of defects at the $\mathrm{Si}-\mathrm{SiO}_{2}$ boundary. After oxidation, Al field electrodes of $1 \mathrm{~mm}$ diameter were deposited on the wafers. At the room temperature, only $\sim 30 \%$ of prepared MDS structures showed noticeable relaxation of $C(t)$ with non-equilibrium depletion within times less than $1 \mathrm{~s}$, the rest MDS structures showed practically no relaxation of $C(t)$ 
within several minutes. It indicates that this nonequilibrium depletion relaxation in $30 \%$ of structures is caused by defects at the boundary $\mathrm{Si}_{-} \mathrm{SiO}_{2}$, which are non-homogeneously distributed in wafers and "cover" $30 \%$ of the area with deposited Al electrodes. There are some grounds to assume that these non-homogeneously distributed defects in $\mathrm{Si}-\mathrm{SiO}$ wafers are related with metal impurity precipitates, which are beneficial to be created at the $\mathrm{Si}-\mathrm{SiO}_{2}$ boundary from the energy viewpoint. These metal precipitates themselves (like to real Si surfaces doped with $\mathrm{Au}$ ) or as a consequence of their interaction with $\mathrm{Si}$ and $\mathrm{SiO}_{2}$ create defects with energy levels in the $\mathrm{Si}$ forbidden gap at the $\mathrm{Si}_{-} \mathrm{SiO}_{2}$ boundary. The defects are efficient centers for majority carriers to be emitted into the respective allowed band in $\mathrm{Si}$. Besides, they are the centers promoting generation of electron-hole pairs with creation of an inversion layer at the silicon surface in the course of its non-equilibrium depletion. The role of these metal impurities at the Si$\mathrm{SiO}_{2}$ boundary was partially analyzed in Chapter 10 of the book [15].

To study the mechanism of acceleration of nonequilibrium depletion relaxation in strong electric fields, we took MDS structures with the relaxation times less or of the order of $1 \mathrm{~s}$ (after $30 \%$ selection). When applying the $\Pi$-pulse depleting voltage $V$ to $\operatorname{MDS}(n)$ capacitor, capacitance relaxation $C(t)$ takes place as a consequence of generation at the boundary with $n$-Si some positive (hole) charge $Q_{p}(t)$ that is located both in $\mathrm{SES}$ of $\mathrm{Si}_{-} \mathrm{SiO}_{2}$ boundary and in v-band as free holes [12]:

$d Q / d t=\left(q \varepsilon \varepsilon_{0} n_{0} C_{d} /(C(t))^{3}\right)[d C(t) / d t]$,

where $\varepsilon$-dielectric permittivity of semiconductor, $\varepsilon_{0}=$ $8.85 \cdot 10^{-12} \mathrm{~F} / \mathrm{m}, C_{d}$ is the capacitance of dielectric $\left(\mathrm{SiO}_{2}\right)$, $n_{0}$ - equilibrium concentration of electrons in $n$-Si bulk, which is determined using the formula [25]:

$n_{0}=\left(2 / q \varepsilon \varepsilon_{0}\right)\left[d V / d\left(1 / C^{2}\right)\right]$.

In experiments, the growth of the value $C(t)$ was always monotonic without any features in the form of additional parts with accelerated relaxation, that is in MDS structures under investigation tunneling of charge carriers from oxide traps does not play any noticeable role as it took place in the aged real surface. The $d Q_{p} / d t$ value was determined in accord with (13) at the moment $t \approx 0$, when the value of the field $E_{s}$ at $n$-Si surface equals [20]:

$E_{s}=Q_{s v} / \varepsilon \varepsilon_{0}=q n_{0} / C_{s v}=q n_{0}\left[1 / C(0)-1 / C_{d}\right]$.

It should be noted that at the very beginning $(t \approx 0)$ after depletion realization, the value $(1 / q) d Q_{p} / d t$ is determined by the velocity of electron emission from SES into c-band of $n$-Si, and then depletion relaxation is realized as a consequence of electron-hole pair generation via SES or (less probable) via impurity levels in SCR. In details, these processes were studied in [3946]. In particular, separation of emission processes and those of generation of electron-hole pairs via SES and levels in SCR was performed in [44] using the change of the initial band caused by an additional external voltage.
It was shown that, as a rule, the emission process of electrons from SES into $n$-Si c-band dominates in the absence of an additional voltage in the vicinity of the initial moment $(t \approx 0)$. It is noteworthy that it is promoted both with a high concentration of SES, as compared to the concentration of levels in SCR, and large cross-sections of capturing electrons in SES as a consequence of their location at the boundary $\mathrm{Si}$ $\mathrm{SiO}_{2}[61]$.

It was found in our investigations that, starting from $E_{s} \geq 1 \cdot 10^{4} \mathrm{~V} / \mathrm{cm}, 1 / q\left(d Q_{p} / d t \sim \exp \left(v E_{s}^{2}\right)\right.$, that is acceleration of relaxation due to high $E_{s}$ values is caused by the Franz-Keldysh effect. As mentioned above, one can write in this case:

$1 / q\left(d Q_{p} / d t\right)=\kappa N_{t} C_{n} \exp \left(-E_{t} / k T+v E_{s}^{2}\right)$,

where $v=(1 / 24)\left((q \hbar)^{2} /\left(m(k T)^{3}\right)\left[1+\left(\left(E_{t}-B\right) k T / \sigma^{2}\right)\right]^{3}\right.$. Notations in (16) are the same as adduced above.

The values calculated for MDS structures was as follows: the energy position of SES relatively to c-band $E_{t}=0.59 \mathrm{eV}$ (as determined from the $(1 / q)\left(d Q_{p} / d t\right)(1 / T)$ dependence); parameter of electron-phonon coupling $\sigma$ changes within limits $0.062-0.053 \mathrm{eV}$, when $T$ changes from 280 up to $370 \mathrm{~K}$. These $\sigma$ values doubly exceed those for real Si surface, which means an essential role of thermal oxide phonons as well as impurity precipitates at the $\mathrm{Si}-\mathrm{SiO}_{2}$ boundary when electrons are emitted from SES into Si c-band.

In [36] we investigated MDS structures that did not show any relaxation of non-equilibrium depletion for several minutes (rest $70 \%$ of structures), but this relaxation appeared after $\gamma$-irradiation of these structures from the ${ }^{60} \mathrm{Co}$ source with doses $D \geq 3 \cdot 10^{4} \mathrm{rad}$. It is known that $\mathrm{Si}_{-} \mathrm{SiO}_{2}$ boundary is the least resistant to radiation in MDS structures [64].

In this work, we investigated acceleration of nonequilibrium depletion relaxation $C(t)$ for $\mathrm{M}-\mathrm{SiO}_{2}-n-\mathrm{Si}$ structures in strong electric fields after stimulation of this relaxation with $\gamma$-irradiation of doses $D=3 \cdot 10^{4}$; $1 \cdot 10^{5} ; 1 \cdot 10^{6} \mathrm{rad}$. As in the case of MDS structures, where relaxation is determined by the presence of precipitate defects at the $\mathrm{Si}-\mathrm{SiO}_{2}$ boundary, MDS structures after $\gamma$-irradiation are characterized by the relation (16), which means that acceleration of relaxation $1 / q\left(d Q_{p} / d t\right)$ possesses thermal-field nature based on the FranzKeldysh effect. For instance, Fig. 21 illustrates the dependences $1 / q\left(d Q_{p} / d t\right)$ on $E_{s}$ and $T^{-1}$ for definite $T$ and $E_{s}$ after $\gamma$-irradiation of the MDS structure with the dose $D=3 \cdot 10^{4} \mathrm{rad}$. With growing $D$, the respective values of $1 / q\left(d Q_{p} / d t\right)$ grow as well. For example, when $T=296 \mathrm{~K}$, $E_{s}=7.7 \cdot 10^{4} \mathrm{~V} / \mathrm{cm}$ and $D=3 \cdot 10^{4}, 1 \cdot 10^{5}, 1 \cdot 10^{6} \mathrm{rad}$, the values of $1 / q\left(d Q_{p} / d t\right)$ were $8 \cdot 10^{11}, 4 \cdot 10^{12}, 1 \cdot 10^{13} \mathrm{~cm}^{-2} \mathrm{~s}^{-1}$, respectively. It should be noted that the $1 / q\left(d Q_{p} / d t\right)$ values at the same $T$ and $E_{s}$ were always less after $\gamma$ irradiation (approximately by an order) than those in MDS structures where relaxation is determined by precipitates at the $\mathrm{Si}-\mathrm{SiO}_{2}$ boundary.

Relaxation of non-equilibrium depletion for above two MDS structures differs by other parameters, too. So, 
after $\gamma$-irradiation of MDS structures we found: $E_{t}=$ $0.54 \mathrm{eV}, \sigma=0.047-0.042 \mathrm{eV}$ for $T=290-370 \mathrm{~K}$, while for the MDS structure with precipitates: $E_{t}=0.59 \mathrm{eV}$, $\sigma=0.062-0.053 \mathrm{eV}$.

Thereof, one can draw a conclusion that the nature of SES, from which electrons are emitted into c-band, is different in these MDS structures. After $\gamma$-irradiation, it is probable broken bonds of silicon $\equiv \mathrm{Si}^{*} \cdot[65]$. Lower $\sigma$ values in MDS structures after irradiation indicate that in the MDS structures with precipitates an essential role is played by phonons of these precipitates, while in the structures after $\gamma$-irradiation essential are the phonons in Si and $\mathrm{SiO}_{2}$.

\section{Ways to neutralize non-equilibrium depletion relaxation in silicon by using majority charge carriers}

Mechanisms of non-equilibrium depletion relaxation considered in previous chapters both for low depleting voltages, when they do not cause acceleration of relaxation, and at medium voltages, when this acceleration is observed, but avalanche multiplication of carriers does not still appear in SCR, indicate that depletion relaxation is determined, first of all, by the SES concentration. SES are the main source of majority charge carrier emission into the respective $\mathrm{Si}$ band, or the centers promoting generation of electron-hole pairs with changing the semiconductor depleted layer by the inversion one. Therefore, to neutralize non-equilibrium depletion relaxation it is necessary by any way to considerably decrease the SES concentration. There offered were several methods to reach this purpose. First, the transfer itself from real surface to the thermally oxidized one decreases considerably (by orders) the SES concentration [1-14]. Besides, the decrease in SES concentration at the boundary $\mathrm{Si}_{-} \mathrm{SiO}_{2}$ (thermal way) takes place as a result of thermal or laser (light way) annealing of the $\mathrm{Si}_{-} \mathrm{SiO}_{2}$ system [65] after its preparation.

SES are considerably neutralized if using "chlorine" technology in the course of silicon oxidation (see, for instance, $[66,67]$ ), when silicon is oxidized in the atmosphere of dry oxygen with addition of $\mathrm{HCl}\left(\mathrm{Cl}_{2}\right)$ or another compound that contains chlorine, or one performs a high-temperature treatment (at $\sim 1100{ }^{\circ} \mathrm{C}$ ) of the $\mathrm{Si}-\mathrm{SiO}_{2}$ system already oxidized in the mixture of dry $\mathrm{O}_{2}$ and $1 \% \mathrm{HCl}$.

Much more efficient damping the centers of nonequilibrium depletion relaxation (SES and defects in SCR) can be reached by combination of chlorine oxidation and the way for gettering the defects by phosphorus diffusion from the rare side of Si wafer [68]. All the enumerated methods provide an essential decrease in the SES concentration and defects in SCR and seem to be rather efficient for decreasing the velocity of non-equilibrium depletion relaxation (with respective times up to tens of minutes).
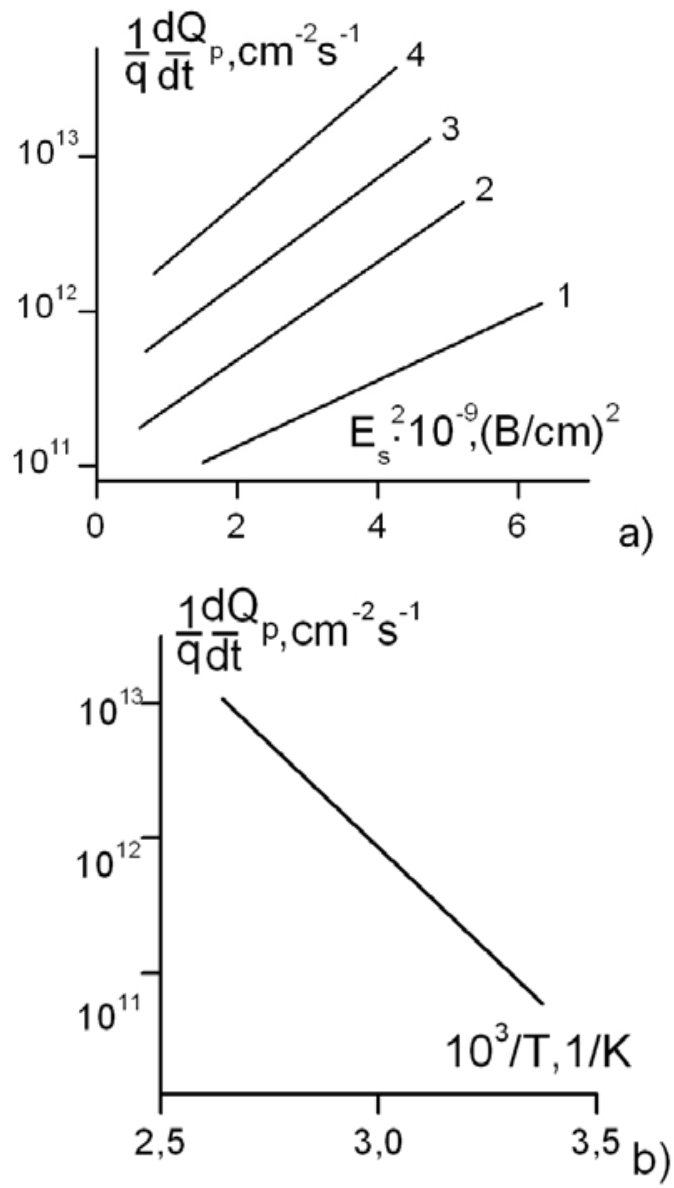

Fig. 22. a) Dependences of the generation velocity for the positive charge $(d Q / d t)$ on $n$-Si surface in MDS structure after $\gamma$-irradiation of it with the dose $3 \cdot 10^{4} \mathrm{rad}(T, \mathrm{~K}: 1-300$, $2-322,3-348,4-365)$. b) Dependence of $d Q / d t$ for $E_{s}=$ $5 \cdot 10^{4} \mathrm{~V} / \mathrm{cm}$ on temperature $(1 / T, \mathrm{~K})$ for $\gamma$-irradiated MDS structures based on $n$-Si ( $4 \mathrm{Ohm} / \mathrm{cm})$.

As to the field MDS structures, we offered a new method for damping the relaxation of non-equilibrium depletion, which enabled to eliminate relaxation of the capacitance $C(t)$ of the MDS structure, when this value changes, by applying a definite depleting voltage $V$ (MDS varicap) [38]. This method is based on a gradual increase of direct (or П-pulse) voltage $V$ up to the value when the avalanche arises in the depleted layer of semiconductor (silicon) that is caused by heating the charge carriers emitted from SES. It results in a local breakdown of $\mathrm{SiO}_{2}$ with creation of a high-ohmic channel to pump out minor charge carriers from silicon, which prevents creation of the inversion layer.

This work was performed using two types of MDS structures prepared like to those described in Chapter 4. Fig. 23 shows volt-farad characteristics $\left(C / C_{d}(V)\right)$ obtained using the MDS structure prepared in accord without the chlorine technology (KEФ-4,5; (100) plane; oxidation in dry oxygen at $1100^{\circ} \mathrm{C}$ for $180 \mathrm{~min}$ with further annealing at $400{ }^{\circ} \mathrm{C}$ for $40 \mathrm{~min}$ in $\mathrm{H}_{2} ; d_{\mathrm{SiO} 2}=$ $1300 \AA$ ). $50 \%$ of prepared MDS structures had no 
relaxation $C / C_{d}(t)$ at the room temperature for several seconds. Given in Fig. 23 are data for structures with the relaxation time less than $1 \mathrm{~s}$. This relaxation is probably caused by emission and generation processes with participation of SES related with defects (precipitates) at the $\mathrm{Si}-\mathrm{SiO}_{2}$ boundary. Immediately after applying the $\Pi$ pulse $\mathrm{V}$, one can observe the dependence $C / C_{d}(V)$ described by the curve 1 (dots). At $V<-15 \mathrm{~V}$, visible relaxation $C / C_{d}(V)$ with the time $t$ (transfer from the curve 1 to the curve 2) begins. The relaxation time depends on the $V$ value and varies from $\sim 1 \mathrm{~s}$ at $V=$ $-15 \mathrm{~V}$ down to $100 \mathrm{~ms}$ at $V<-70 \mathrm{~V}$. With increasing the value $|V|$, for example up to $V=-140 \mathrm{~V}\left(t_{\text {імр }} \sim 5 \mathrm{~s}\right)$, the avalanche arises in SCR, and a high-ohmic conductive channel forms from material of field (metal) electrode $\left(\mathrm{SiO}_{2}\right.$ breakdown). Initiation of the avalanche and $\mathrm{SiO}_{2}$ breakdown were fixed by a jump-like increase in the value $C / C_{d}(V)$ up to unity. Calculations show that the field $E_{s}$ reaches the value $6 \cdot 10^{5} \mathrm{~V} / \mathrm{cm}$ at this moment, which agrees well with the data of works $[49,50]$ where appearance of carrier avalanche was considered. The breakdown regime was kept not longer than $5 \mathrm{~s}$ for the MDS structure to be not destroyed by heating (with significant spreading the channel and changes in $C(t)$ values at $V \approx 0$ ). As it was shown in [69], the channel formed for tens of nanoseconds has the diameter of 0.2 $1 \mu \mathrm{m}$. Repeated measurements of the dependences $C / C_{d}(V)$ after formation of the conductive channel in $\mathrm{SiO}_{2}$ resulted in the curve 3 at $V<-140 \mathrm{~V}$, the values $C / C_{d}(V)$ of which were not changed with time $t$, that is the curve 3 did not convert to the curve 2 . The curve 3 was kept unchanged in measurements with "formed" MDS structures for 3 months.

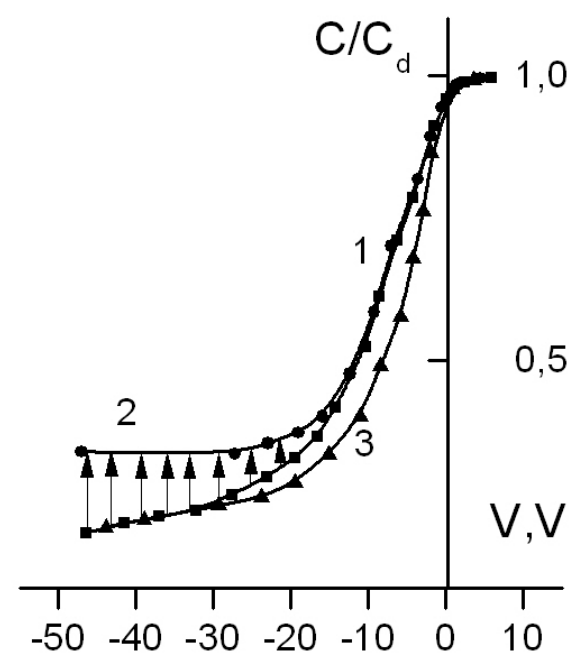

Fig. 23. Dependences of relative capacitance $\left(C / C_{d}\right)$ relaxation in MDS structures created by dry thermal oxidation of silicon (КЕФ-4,5) on the voltage on the metal electrode of the MDS structure: 1 - initial $C / C_{d}$ values before their relaxation with time after applying the external voltage $V ; 2-C / C_{d}$ values after their relaxation with time; $3-C / C_{d}$ values (stable in time) after creation of the conductive channel inside dielectric.

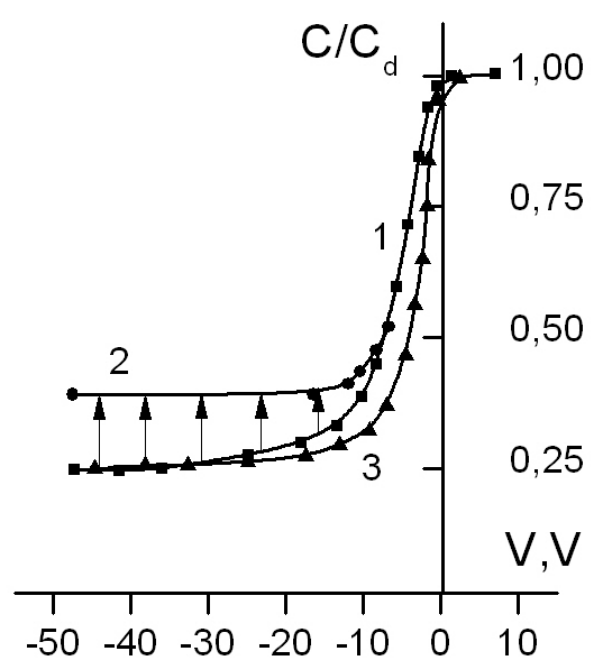

Fig. 24. Dependence on the voltage $V$ for capacitance $\left(C / C_{d}\right)$ relaxation in the MDS structure with oxide thermally grown using the chlorine technology after $\gamma$-irradiation of the structure with the dose $D=3 \cdot 10^{4} \mathrm{rad}$ : $1-$ initial $C / C_{d}$ values before their relaxation with time; $2-C / C_{d}$ value after finishing relaxation with time; $3-C / C_{d}$ values (stable in time) after creation the conductive channel in dielectric.

Depicted in Fig. 24 are the similar dependences $C / C_{d}(V)$ for the structure prepared using the chlorine technology. As mentioned above, $70 \%$ of these MDS structures had no visible relaxation of non-equilibrium depletion (or their relaxation times were of the order of several minutes at the room temperature). The appearance of non-equilibrium depletion relaxation (Fig. 24) was caused by $\gamma$-irradiation of the MDS structures $\left({ }^{60} \mathrm{Co}, D=5 \cdot 10^{4} \mathrm{rad}\right)$. It is seen from Fig. 24 that visible relaxation with time (transfer from the curve 1 (dots) to the curve 2) begins already at $V<-8 \mathrm{~V}$, that is for lower voltages $|V|$ than those in the MDS structure considered above. When $V<-130 \mathrm{~V}$, the carrier avalanche arose in SCR of semiconductor, and a high-ohmic conductive channel formed in $\mathrm{SiO}_{2}$ with the cross-section considerably less than the area of the MDS structure $\left(\sim 1 \mathrm{~mm}^{2}\right)$. As earlier, for the structure considered in Fig. 23, it provides keeping the curve 3 that does not convert to the curve 2 when $V>-130 \mathrm{~V}$. The absence of relaxation for the curves 3 with time is caused by extraction of minor charge carriers from SCR through the formed channel in $\mathrm{SiO}_{2}$ into the metal electrode. In this case, the inversion layer in SCR of $n-\mathrm{Si}$ was not formed, and the measured capacitance $C(V, t)$ depends only on the $V$ value.

\section{Conclusions}

Works devoted to studying the mechanisms responsible for acceleration of non-equilibrium depletion relaxation in silicon under strong electric fields and considered in this paper indicate that there exist several mechanisms for field acceleration of relaxation in dependence on the 
physical-and-chemical state of the investigated surface. As a rule, immediately after creation of non-equilibrium depletion by an external voltage $V$ when the electric fields on silicon surface reach the values $E_{s}=3 \cdot 10^{3}$ $1 \cdot 10^{5} \mathrm{~V} / \mathrm{cm}$, acceleration of relaxation by the field $E_{s}$ begins. This acceleration can be caused by the Frenkel and Franz-Keldysh effects. In the first case, acceleration is caused by lowering the barrier for the transition of a charge carrier from SES (with a far-acting potential) into the respective allowed band of majority carriers; in the second one, the transition of a carrier from SES is accelerated by creation of virtual states in the allowed band of silicon under high $E_{s}$ values (as a rule, higher than in the Frenkel effect). In both cases, these transitions of carriers have thermal-and-field nature and depend on interaction of carriers with phonons of the medium around SES.

Transitions of carriers from SES are the main reason of non-equilibrium depletion relaxation. In thermally oxidized silicon, where the SES concentration can be decreased by an order, SES can also play a role of centers promoting generation of electron-hole pairs, when the depleted layer of SCR is changed with the inversion layer of minor carriers. Generation via local centers in SCR is possible but in most cases is nonefficient, as the role of SES due to their high concentration and a large cross-section for capturing the carriers appears to be dominant.

Charge carriers that passed from SES into allowed bands are accelerated with increasing voltage in $\mathrm{SCR}$ ( $E_{s}$ grows), which causes the avalanche due to impact generation of electron-hole pairs, which, with further growth of the voltage, can result in breakdown of $\mathrm{SiO}_{2}$ in the MDS structure.

Finally, it is noteworthy that in some cases (real aged silicon surface; real surface, doped with $\mathrm{Zn}$ impurity) one can observe relaxation acceleration by the field in some time after its origination, when charge carriers begin to pass from traps in oxide (or silicate) film into allowed bands of silicon.

\section{References}

1. W. Shockly, Electrons and Holes in Semiconductors. Willey, N.Y., 1950.

2. Semicoductor Surface Physics, Ed. by R.H. Kingston. Willey, Phyladelphia, 1957.

3. A. Many, Y. Goldstein, N.B. Crover, Semiconductor Surfaces. John Willey and Sons, NY., 1965.

4. D.R. Frankl, Electrical Properties of Semiconductor Surface. Pergamon Press NY, 1967.

5. V.I. Lyashenko, V.G. Litovchenko, I.I. Stepko, V.I. Stricha, L.V. Lyashenko, Electron Properties on Semiconductor Surface. Naukova dumka, Kiev, 1968 (in Russian).

6. A.V. Rzhanov, Electron Processes on Semiconductor Surface. Nauka, Moscow, 1971 (in Russian).
7. R.O. Litvinov, Effect of Surface on Characteristic of Semiconductor Devices. Naukova dumka, Kiev, 1972 (in Russian).

8. S.M. Sze, Physics of Semiconductor Devices. John Willey and Sons, 1981.

9. W. Shockly, G.L. Pearson, Modulation of conductance of thin film of semiconductors by surface charge // Phys. Rev. 74, p. 232-236 (1948).

10. M.M. Attala, E. Tannenbaum, E.E. Schebner, Stabilization of silicon surfaces by thermally grown oxides // Bell. Syst. Techn. J. 30(5), p. 749-765 (1959).

11. D. Kahng, M.M. Attala, $\mathrm{Si}-\mathrm{SiO}_{2}$ field induced surface device // IRE Solid State Dev. Res. Conf., Pittsburg, 1960.

12. V.G. Litovchenko, A.P. Gorban', Physics of Microelectronic System Metal-DielectricSemiconductor. Naukova dumka, Kiev, 1978 (in Russian).

13. B.A. Nesterenko, O.V. Snitko, Physical Properties of Atomically Clean Semiconductor Surface. Naukova dumka, Kiev, 1983 (in Russian).

14. V.A. Zuev, A.V. Sachenko, K.B. Tolpygo, Nonequilibrium Processes in Semiconductors and Semiconductor Devices. Sovetskoye radio, Moscow, 1977 (in Russian).

15. V.E. Primachenko, O.V. Snitko, Physics of the Semiconductor Surface Doped with Metals. Naukova dumka, Kiev, 1988 (in Russian).

16. V.S. Vavilov, V.F. Kiseljov, B.N. Mukashev, Defects in Silicon and on its Surface. Nauka, Moscow, 1990 (in Russian).

17. C.G. Garret, W.H. Brattain, Physical theory of semiconductor surface // Phys. Rev. 99(2), p. 376397 (1955).

18. J.R. Schriffer, Effective mobility of carriers in the surface SCR // Phys. Rev. 97(3), p. 641-652 (1955).

19. V.E. Primachenko, O.V. Snitko, V.V. Milenin, Investigation of non-equilibrium depletion effect in silicon with majority charge carriers // Ukrains 'kyi fizychnyi zhurnal 10(4), p. 382-388 (1965) (in Ukrainian).

20. V.E. Primachenko, O.V. Snitko, V.V. Milenin, On the mechanism of non-equilibrium depletion effect in silicon with majority charge carriers // Ibid. p. 389-397.

21. V.E. Primachenko, O.V. Snitko, V.V. Milenin, Nonequilibrium field effect on $\mathrm{Si}$ in the region of high depletion // Phys. status solidi 11(3), p. 711718 (1965).

22. G. Rupprecht, Cross-section of midgap surface states in silicon by pulsed field effect experiments // J. Phys. Chem. Solids 14(1), p. 208-215 (1960).

23. A.E. Yunovich, V.I. Tikhonov, On kinetics of surface phenomena in silicon, In: Surface Properties of Semiconductors. Published by AS USSR, Moscow, 1962, p. 127-137 (in Russian).

24. A. Many, N.B. Grover, Y. Roldstein, E.'Harnik, 
a) Surface mobility measurements in germanium $/ / \mathrm{J}$. Phys. Chem. Solids, 14, p. 186-192 (1960).

b) Fast surface states in germanium at low temperatures // Ibid. p. 193-199 (1960).

25. V.V. Antoshchuk, V.V. Milenin, V.E. Primachenko, O.V. Snitko, Determination of some silicon parameters at $77 \mathrm{~K} / /$ Fizika i tekhnika poluprovodnikov 10(10), p. 2004-2006 (1976) (in Russian).

26. A.I. Klimovskaya, Subsurface repopulation of valleys in electron silicon, In: Problems of Physics of Semiconductor Surface. Naukova dumka, Kiev, 1981, p. 179-202 (in Russian).

27. V.E. Primachenko, Investigation of electron properties of germanium and silicon surface. Abstract of the thesis for candidate of sciences degree (physics and mathematics). The Institute of Semiconductor Physics, NAS of Ukraine, Kiev, 1965 (in Russian).

28. W.S. Boyle, G.E. Smith, Charge coupled semiconductor devices // Bell. Syst. Techn. J. 49(4), p. 587-593 (1970).

29. G.F. Amelio, M.P. Tompsett, G.E. Smith, Experimental verification of the charge coupled // Bell. Syst. Techn. J. 49(4), p. 593-600 (1970).

30. S.I. Kirillova, V.E. Primachenko, O.V. Snitko, Acceleration of non-equilibrium depletion relaxation on the real silicon surface in strong electric fields // Fizika i tekhnika poluprovodnikov 15(5), p. 874-880 (1981) (in Russian).

31. S.I. Kirillova, V.E. Primachenko, O.V. Snitko, Influence of oxidation on non-equilibrium depletion relaxation on silicon surface // Poverkhnost': Fizika, khimiya, mekhanika 116, p. 83-86 (1982) (in Russian).

32. S.I. Kirillova, V.E. Primachenko, O.V. Snitko, Nonequilibrium depletion relaxation on real surface of silicon doped with gold // Ukrainskii fizicheskii zhurnal 27(1), p. 54-59 (1982) (in Russian).

33. S.I. Kirillova, V.E. Primachenko, O.V. Snitko, Non-equilibrium depletion relaxation on silicon surface doped with zinc // Ukrainskii fizicheskii zhurnal 27(8), p. 1181-1186 (1982) (in Russian).

34. S.I. Kirillova, V.E. Primachenko, O.V. Snitko, O.S. Frolov, Non-equilibrium depletion relaxation on silicon MDS structures in strong electric fields // Mikroelektronika 13(3), p. 236-242 (1983) (in Russian).

35. A.I. Shkrebtii, S.I. Kirillova, V.E. Primachenko, O.V. Snitko, Non-equilibrium depletion relaxation in electric field non-homogeneity caused by islandlike metal inclusions on silicon surface // Ukrainskii fizicheskii zhurnal 30(6), p.937-940 (1985) (in Russian).

36. S.I. Kirillova, V.E. Primachenko, O.V. Snitko, Non-equilibrium depletion relaxation in $\gamma$ irradiated silicon MDS structures in strong electric fields // Ukrainskii fizicheskii zhurnal 30(1), p. 150-154 (1985) (in Russian).
37. S.I. Kirillova, V.E. Primachenko, O.V. Snitko, Nonequilibrium depletion relaxation in strong electric fields under various conditions at the silicon surface // Phys. status solidi (a) 88(2), p. 647-654 (1985).

38. V.E. Primachenko, S.I. Kirillova, O.V. Snitko, O.S. Frolov, Non-relaxating varactor. Authors' certificate № 1258189 from 15 May 1986 (in Russian).

39. M. Zerbst, Relaxation Effekte on HalbleiterIsolate-Grenzflachen //Z. angew. Phys. 22(1), p. 30-33 (1966).

40. F.P. Heiman, On the determination of minority carrier lifetime from the transient response of MOS capacitor //IEEE, Trans. Electron. Devices, ED-14 (11), p. 781-784 (1967).

41. J.S.I. Huang, Bulk lifetime determination using MOS-capacitor // Proc. IEEE 58(11), p. 1848-1850 (1970).

42. J. Müller, B. Schick, Transient responses of a pulsed MIS-capacitor // Solid-State Electron. 13(8), p. 1319-1332 (1970).

43. D.K. Schroder, J. Guldberg, Interpretation of surface and bulk effects using the pulsed MIS capacitor // Solid-State Electron. 14(12), p. 1285-1297 (1971).

44. L.S. Wei, J.G. Simmons, Transient emission and generation currents in MIS capacitors // Solid-State Electron. 18(10), p. 853-857 (1975).

45. R.S. Nakhmanson, Theory of the surface capacitance // Fizika tverdogo tela 6(4), p. 11151123 (1964) (in Russian).

46. A.P. Gorban', N.L. Dmitruk, V.G. Litovchenko, O.I. Maeva, B.N. Romanyuk, Generation of charge carriers in semiconductor structures based on $\mathrm{Si}$ and GaAs with non-equilibrium depletion layer // Poluprovodnikovaya tekhnika $i$ mikroelektronika N 28, p. 86-95 (1978) (in Russian).

47. N.L. Dmitruk, V.I. Lyashenko, O.I. Maeva, Investigation of non-equilibrium capacitance relaxation in MDS structures based on wide-band semiconductors, In: Electron Processes on Surface of Semiconductors and at their Boundary with Dielectric. Nauka, Novosibirsk, 1974, p. 243-247 (in Russian).

48. O.V. Snitko, M.K. Sheinkman, Kh.E. Azimkhodzhaev, G.N. Golynnaya, Investigation of the field effect in CdS single crystals, In: Electron Processes on Surface and in Monocrystalline Layers of Semiconductors. Nauka, Novosibirsk, p. 136-143 (1967) (in Russian).

49. A. Goetzberger, E.H. Nicollian, Avalanche and tunneling effects in MOS structures // J. Appl. Phys. 38(12), p. $4582-4590$ (1967).

50. A. Goetzberger, E.H. Nicollian, Transient voltage breakdown due to avalanche in MIS capacitors // Appl. Phys. Lett. 9 (12), p. 444-446 (1967).

51. S.F. Timashev, On thermal ionization of deep centers in SCR of semiconductors // Fizika tverdogo tela 14(1), p. 171-174 (1972) (in Russian). 
52. S.F. Timashev, On the Frenkel' effect in thermaland-field ionization of deep centers // Fizika $i$ tekhnika poluprovodnikov 8(4), p. 804-806 (1974) (in Russian).

53. V.A. Gritsenko, Kinetics of non-equilibrium processes caused by the Frenkel' effect in strong electric field, In: Electron Processes on Surface and Boundary of Semiconductors. Nauka, Novosibirsk, 1974, p. 115-118 (in Russian).

54. W. Franz, Einflu $\beta$ eines elektrischen Feldes auf eine optische Absorptionskonte // Zeits. Naturforschung 13a (2), p. 484-490 (1958).

55. L.V. Keldysh, On the influence of crystal lattice vibrations on bearing the electron-hole pairs in strong electric field // Zhurnal eksperimental'noi $i$ teoreticheskoi fiziki 34(4), p. 962-968 (1958) (in Russian).

56. V. Karpus, Influence of electron-phonon interaction on ionization of deep centers with strong electric field // Pis'ma $v$ Zhurnal eksperimental'noi $i$ teoreticheskoi fiziki 44(7), p. 334-336 (1986) (in Russian).

57. V.V. Murina, Yu. F. Novototskii-Vlasov, A.S. Petrov et al., Investigation of properties of real silicon surface, In: Electron Processes on Surface of Semiconductors. Nauka, Novosibirsk, 1974, p. 262266 (in Russian).

58. V.V. Milenin, V.I. Poludin, V.E. Primachenko, O.V. Snitko, Influence of Ag and Au impurities on electron properties of silicon // Ukrainskii fizicheskii zhurnal 20(4), p. 616-620 (1975) (in Russian).

59. V.V. Milenin, V.E. Primachenko, G.A. Sukach, Recombination processes at laser excitation of $\mathrm{Si}$ surface doped with Au // Ukrainskii fizicheskii zhurnal 21(8), p. 1301-1304 (1976) (in Russian).

60. V.V. Milenin, V.I. Poludin, V.E. Primachenko et al., Creation of $\mathrm{Au}$ and $\mathrm{Ag}$ disperse structures in the course of their adsorption on $\mathrm{Si}$ surface //
IIzvestiya AN SSSR, Neorganicheskiye materialy 13(3), p. 515-517 (1977) (in Russian).

61. A.A. Pakhomov, I.N. Yassiyevich, Influence of the boundary on capture and emission of carriers by deep centers // Fizika i tekhnika poluprovodnikov 27(3), p. 482-487 (1993) (in Russian).

62. V.A. Gurtov, Influence of ionizing radiation on properties of MDS devices // Obzory po elektronnoi tekhnike. Ser. 2, 14, p. 1-31 (1978) (in Russian).

63. A.B. Gerasimov, N.R. Aigina, L.I. Ushenashvili, A.G. Shilo // Zarubezhnaya elektronnaya tekhnika 1, p. 1-47 (1979) (in Russian).

64. V.Ya. Kiblik, R.O. Litvinov, V.G. Litovchenko, N.M. Litovchenko, Influence of $\gamma$-irradiation on generation-recombination characteristics of MDS structures // Ukrainskii fizicheskii zhurnal 22(7), p. 1097-1190 (1977) (in Russian).

65. V.E. Primachenko, S.I. Kirillova, V.A. Chernobai, E.F. Venger, Electron states at $\mathrm{Si}-\mathrm{SiO}_{2}$ boundary (Review) // Semiconductor Physics, Quantum Electronics and Optoelectronics 8(4), p. 38-54 (2005).

66. R.J. Kriegler, J.C. Cheng, D.R. Colton, The effect of $\mathrm{HCl}$ and $\mathrm{Cl}_{2}$ at the thermal oxidation // J. Electrochem. Soc. 119 (31), p. 388 (1972).

67. R.S. Ronien, P.H. Robinson, Hydrogen chloride and chlorine gettering // J. Electrochem. Soc. 119(6), p. 747 (1972).

68. J.W. Swart, E .Charry, Achievment of high lifetime by a combination of $\mathrm{HCl}$ oxidation and $\mathrm{POGO}$ getting techniques //J. Electrochem. Soc. 128(6), p. 1383 (1981).

69. G.V. Stepanov, O.F. Shevchenko, N.S. Muksimov, A.Ye. Lukjanov, I.F. Urazgil'din, Ye.K. Krokhina, Investigation of electrode material mass-transfer on silicon surface and inside $\mathrm{SiO}_{2}$ bulk // Poverkhnost': Fizika, khimiya, mekhanika N 4, p. 79-81 (1983) (in Russian). 